\title{
Query-time Entity Resolution
}

Indrajit Bhattacharya

IBM India Research Laboratory

Vasant Kunj, New Delhi 110 070, India

Lise Getoor

Department of Computer Science

University of Maryland, College Park, MD 20742 USA
INDRAJBH@IN.IBM.COM

GETOOR@CS.UMD.EDU

\begin{abstract}
Entity resolution is the problem of reconciling database references corresponding to the same real-world entities. Given the abundance of publicly available databases that have unresolved entities, we motivate the problem of query-time entity resolution: quick and accurate resolution for answering queries over such 'unclean' databases at query-time. Since collective entity resolution approaches - where related references are resolved jointly - have been shown to be more accurate than independent attribute-based resolution for off-line entity resolution, we focus on developing new algorithms for collective resolution for answering entity resolution queries at query-time. For this purpose, we first formally show that, for collective resolution, precision and recall for individual entities follow a geometric progression as neighbors at increasing distances are considered. Unfolding this progression leads naturally to a two stage 'expand and resolve' query processing strategy. In this strategy, we first extract the related records for a query using two novel expansion operators, and then resolve the extracted records collectively. We then show how the same strategy can be adapted for query-time entity resolution by identifying and resolving only those database references that are the most helpful for processing the query. We validate our approach on two large real-world publication databases where we show the usefulness of collective resolution and at the same time demonstrate the need for adaptive strategies for query processing. We then show how the same queries can be answered in real-time using our adaptive approach while preserving the gains of collective resolution. In addition to experiments on real datasets, we use synthetically generated data to empirically demonstrate the validity of the performance trends predicted by our analysis of collective entity resolution over a wide range of structural characteristics in the data.
\end{abstract}

\section{Introduction}

With the growing abundance of publicly available data in digital form, there has been intense research on data integration. A critical component of the data integration process is the entity resolution problem, where uncertain references in the data to real-world entities such as people, places, organizations, events, etc., need to be resolved according to their underlying real-world entities. Entity resolution is needed in order to solve the 'deduplication' problem, where the goal is to identify and consolidate pairs of records or references within the same relational table that are duplicates of each other. It also comes up as the 'fuzzy match' problem, where tuples from two heterogeneous databases with different keys, and possibly different schemas, need to be matched and consolidated. It goes by different 
names even within the data mining and database communities, including record linkage, object consolidation, and reference reconciliation.

The problem has a long history, and recent years have seen significant and fruitful research on this problem. However, in spite of the widespread research interest and the practical nature of the problem, many publicly accessible databases remain unresolved, or partially resolved, at best. The popular publication databases, CiteSeer and PubMed, are representative examples. CiteSeer contains several records for the same paper or author, while author names in PubMed are not resolved at all. This is due to a variety of reasons, ranging from rapid and often uncontrolled growth of the databases and the computational and other expenses involved in maintaining resolved entities.

Yet, millions of users access and query such databases everyday, mostly seeking information that, implicitly or explicitly, requires knowledge of the resolved entities. For example, we may query the CiteSeer database of computer science publications looking for books by 'S Russell' (Pasula, Marthi, Milch, Russell, \& Shpitser, 2003). This query would be easy to answer if all author names in CiteSeer were correctly mapped to their entities. But, unfortunately, this is not the case. According to CiteSeer records, Stuart Russell and Peter Norvig have written more than 100 different books together. One of the main reasons behind databases containing unresolved entities is that entity resolution is generally perceived as an expensive process for large databases. Also, maintaining a 'clean' database requires significant effort to keep pace with incoming records. Alternatively, we may be searching different online social network communities for a person named 'Jon Doe'. In this case, each online community may individually have records that are clean. Even then, query results that return records from all of the sources aggregated together may have multiple representations for the same 'Jon Doe' entity. Additionally, in both cases, it is not sufficient to simply return records that match the query name, 'S. Russell' or 'Jon Doe' exactly. In order to retrieve all the references correctly, we may need to retrieve records with similar names as well, such as 'Stuart Russel' or 'John Doe'. And, most importantly, for the results to be useful, we need to partition the records that are returned according to the real-world entities to which they correspond. Such on-the-fly partitioning of returned results is also necessary when accessing third-party or external databases that do not provide full access possibly due to privacy and other concerns, and can be accessed only via specific query interfaces.

In this paper, we propose an alternative solution for answering entity resolution queries, where we obviate the need for maintaining resolved entities in a database. Instead, we investigate entity resolution at query-time, where the goal is to enable users to query an unresolved or partially resolved database and resolve the relevant entities on the fly. A user may access several databases everyday and she does not want to resolve all entities in every database that she queries. She only needs to resolve those entities that are relevant for a particular query. For instance, when looking for all books by 'Stuart Russell' in CiteSeer, it is not useful to resolve all of the authors in CiteSeer. Since the resolution needs to be performed at query-time, the requirement is that the resolution process needs to be quick, even if it is not entirely accurate.

Though entity resolution queries have not been addressed in the literature, there has been significant progress on the general entity resolution problem. Recent research has focused on the use of additional relational information between database references to improve 
resolution accuracy (Bhattacharya \& Getoor, 2004; Singla \& Domingos, 2004; Dong, Halevy, \& Madhavan, 2005; Ananthakrishna, Chaudhuri, \& Ganti, 2002; Kalashnikov, Mehrotra, \& Chen, 2005). This improvement is made possible by resolving related references or records jointly, rather than independently. Intuitively, this corresponds to the notion that figuring out that two records refer to the same underlying entity may in turn give us useful information for resolving other record pairs that are related. Imagine that we are trying to decide if two authors 'Stuart Russell' and 'S Russell' are the same person. We can be more confident about this decision if we have already decided that their co-authors 'Peter Norvig' and 'P. Norvig' are the same person.

As others have done, in our earlier work (Bhattacharya \& Getoor, 2004, 2007), we have demonstrated using extensive experiments on multiple real and synthetic datasets that collective resolution significantly improves entity resolution accuracy over attribute-based and naive relational baselines. However, its application for query-time entity resolution is not straight-forward, and this is precisely the problem that we focus on in this paper. The first difficulty is that collective resolution works for a database as a whole and not for a specific query. Secondly, the accuracy improvement comes at a considerable computation cost arising from the dependencies between related resolutions. This added computational expense makes its application in query-time resolution challenging.

In this paper, which builds on and significantly extends the work presented in Bhattacharya, Licamele, and Getoor (2006), we investigate the application of collective resolution for queries. First, we formally analyze how accuracies of different decisions in collective resolution depend on each other and on the structural characteristics of the data. The recursive nature of the dependency leads naturally to a recursive 'expand and resolve' strategy for processing queries. The relevant records necessary for answering the query are extracted by a recursive expansion process and then collective resolution is performed only on the extracted records. Using our analysis, we show that the recursive expansion process can be terminated at reasonably small depths for accurately answering any query; the returns fall off exponentially as neighbors that are further away are considered.

However, the problem is that this unconstrained expansion process can return too many records even at small depths; and thus the query may still be impossible to resolve in real-time. We address this issue using an adaptive strategy that only considers the most informative of the related records for answering any query. This significantly reduces the number of records that need to be investigated at query time, but, most importantly, does not compromise on the resolution accuracy for the query.

Our specific contributions in this paper are as follows:

1. First, we motivate and formulate the problem of query-time entity resolution. Our entity resolution approach is based on a relational clustering algorithm. To the best of our knowledge, clustering based on queries in the presence of relations has received little attention in the literature.

2. For collective resolution using relational clustering, we present an analysis of how the accuracy of different resolution decisions depends on each other and on the structural characteristics of the data. We introduce the notion of precision and recall for individual entities, and show how they follow a geometric progression as neighbors at increasing distances are considered and resolved. Our analysis shows that collective 
use of relationships can sometimes hurt entity resolution accuracy. This has not been previously reported in the literature. Our analysis additionally demonstrates the convergent nature of resolution performance for the recursive query-resolution strategy that we propose.

3. For resolving queries collectively, we propose a two-phase 'expand and resolve' algorithm. It first extracts the related records for a query using two novel expansion operators, and then resolves the query by only considering the extracted records. We then improve on this algorithm using an adaptive approach that selectively considers only the 'most informative' ones among the related records for a query. This enables collective resolution at query-time without compromising on resolution accuracy for the query.

4. We present experimental results on two large real-world datasets where our strategy enables collective resolution in seconds. We compare against multiple baselines to show that the accuracy achieved using collective query resolution is significantly higher than those achieved using traditional approaches.

5. We also use synthetically generated data to demonstrate the gains of collective query resolution over a wide range of attribute and relational characteristics. We additionally show that the empirical results are in agreement with the trends predicted by our analysis of collective resolution.

The rest of the paper is organized as follows. In Section 2, we formalize the relational entity resolution problem and entity resolution queries, and also illustrate these with an example. In Section 3, we briefly review the relational clustering algorithm that we employ for collective entity resolution and then, in Section 4, investigate how resolution accuracy for related entities depend on each other for collective resolution using this algorithm. In Section 5, we extend collective resolution for queries, and describe and analyze an unconstrained recursive strategy for collectively resolving a query. We then modify this approach in Section 6 and present our adaptive algorithm that extracts only the 'most informative' references for resolving a query. We present experimental results on real and synthetic data in Section 7, review related work in Section 8 and finally conclude in Section 9.

\section{Entity Resolution and Queries: Formulation}

In this section, we formally introduce the entity resolution problem and also entity resolution queries, and illustrate them using a realistic example - that of resolving authors in a citation database such as CiteSeer or PubMed.

In the simplest formulation of the entity resolution problem, we have a collection of references, $\mathcal{R}=\left\{r_{i}\right\}$, with attributes $\left\{\mathcal{R} . A_{1}, \ldots, \mathcal{R} . A_{k}\right\}$. Let $\mathcal{E}=\left\{e_{j}\right\}$ be the unobserved domain entities. For any particular reference $r_{i}$, we denote the entity to which it maps as $E\left(r_{i}\right)$. We will say that two references $r_{i}$ and $r_{j}$ are co-referent if they correspond to the same entity, $E\left(r_{i}\right)=E\left(r_{j}\right)$. Note that in the case of an unresolved database, this mapping $E(\mathcal{R})$ is not provided. Further, the domain entities $\mathcal{E}$ and even the number of such entities is not known. However, in many domains, we may have additional information about relationships between the references. To model relationships in a generic way, we use 


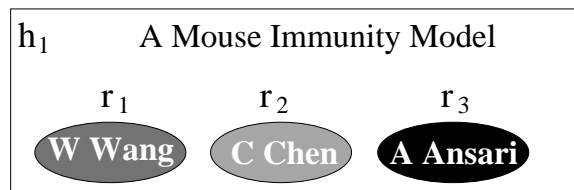

$\mathrm{h}_{3} \quad$ Measuring Protien-bound Fluxetine

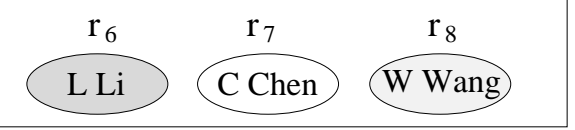

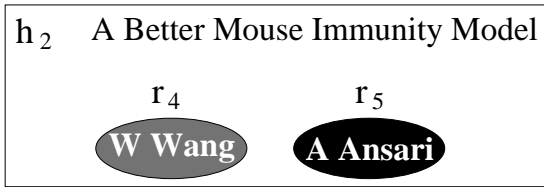

$\mathrm{h}_{4}$ Autoimmunity in Biliary Cirrhosis

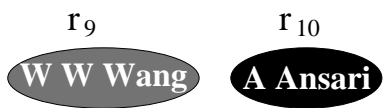

Figure 1: An example set of papers represented as references connected by hyper-edges. References are represented as ovals shaded according to their entities. Each paper is represented as a hyper-edge (shown as a rectangle) spanning multiple references.

a set of hyper-edges $\mathcal{H}=\left\{h_{i}\right\}$. Each hyper-edge connects multiple references. To capture this, we associate a set of references $h_{i} . R$ with each hyper-edge $h_{i}$. Note that each reference may be associated with zero or more hyper-edges.

Let us now look at a sample domain to see how it can be represented in our framework. Consider a database of academic publications similar to DBLP, CiteSeer or PubMed. Each publication in the database has a set of author names. For every author name, we have a reference $r_{i}$ in $\mathcal{R}$. For any reference $r_{i}, r_{i}$.Name records the observed name of the author in the publication. In addition, we can have attributes such as $\mathcal{R}$.Email to record other information for each author reference that may be available in the paper. Now we come to the relationships for this domain. All the author references in any publication are connected to each other by a co-author relationship. This can be represented using a hyper-edge $h_{i} \in \mathcal{H}$ for each publication and by having $r_{j} \in h_{i} . R$ for each reference $r_{j}$ in the publication. If publications have additional information such as title, keywords, etc, they are represented as attributes of $\mathcal{H}$.

To illustrate, consider the following four papers, which we will use as a running example:

1. W. Wang, C. Chen, A. Ansari, "A mouse immunity model"

2. W. Wang, A. Ansari, "A better mouse immunity model"

3. L. Li, C. Chen, W. Wang, "Measuring protein-bound fluxetine"

4. W. W. Wang, A. Ansari, "Autoimmunity in biliary cirrhosis"

To represent them in our notation, we have 10 references $\left\{r_{1}, \ldots, r_{10}\right\}$ in $\mathcal{R}$, one for each author name, such that $r_{1} . N a m e=$ 'W Wang', etc. We also have 4 hyper-edges $\left\{h_{1}, \ldots, h_{4}\right\}$ in $\mathcal{H}$, one for each paper. The first hyper-edge $h_{1}$ connects the three references $r_{1}, r_{2}$ and $r_{3}$ corresponding to the names 'W. Wang', 'C. Chen' and 'A. Ansari'. This is represented pictorially in Figure 1.

Given this representation, the entity resolution task is defined as the partitioning or clustering of the references according to the underlying entity-reference mapping $E(\mathcal{R})$. Two references $r_{i}$ and $r_{j}$ should be assigned to the same cluster if and only if they are 
coreferent, i.e., $E\left(r_{i}\right)=E\left(r_{j}\right)$. To illustrate, assume that we have six underlying entities for our example. This is illustrated in Figure 1 using a different shading for each entity. For example, the 'Wang's of papers 1, 2 and 4 are names of the same individual but the 'Wang' from paper 3 is a reference to a different person. Also, the 'Chen's from papers 1 and 3 are different individuals. Then, the correct entity resolution for our example database with 10 references returns 6 entity clusters: $\left\{\left\{r_{1}, r_{4}, r_{9}\right\},\left\{r_{8}\right\},\left\{r_{2}\right\},\left\{r_{7}\right\},\left\{r_{3}, r_{5}, r_{10}\right\},\left\{r_{6}\right\}\right\}$. The first two clusters correspond to two different people named 'Wang', the next two to two different people named 'Chen', the fifth to 'Ansari' and the last to 'Li'.

Any query to a database of references is called an entity resolution query if answering it requires knowledge of the underlying entity mapping $E(\mathcal{R})$. We consider two different types of entity resolution queries. Most commonly, queries are specified using a particular value $a$ for an attribute $\mathcal{R}$. $A$ of the references that serves as a 'quasi-identifier' for the underlying entities. Then the answer to the query $Q(R . A=a)$ should partition or group all references that have $r . A=a$ according to their underlying entities. For references to people, the name often serves as a weak or noisy identifier. For our example bibliographic domain, we consider queries specified using R.Name. To retrieve all papers written by some person named 'W. Wang', we issue a query using $\mathcal{R}$.Name and 'W. Wang'. Since names are ambiguous, treating them as identifiers leads to undesirable results. In this case, it would be incorrect to return the set $\left\{r_{1}, r_{4}, r_{8}\right\}$ of all references with name 'W Wang' as the answer to our query. This answer does not indicate that $r_{8}$ is not the same person as the other two. Additionally, the answer should include the reference $r_{9}$ for 'W W Wang', that maps to the same entity as the author of the first paper. Therefore, the correct answer to the entity resolution query on 'W Wang' should be the partition $\left\{\left\{r_{1}, r_{4}, r_{9}\right\},\left\{r_{8}\right\}\right\}$.

Entity resolution queries may alternatively be specified using a specific reference. Imagine a CiteSeer user looking at a paper that contains some author name. The user may be interested in looking up other papers written by the same author, even though they may not know who that author is precisely. Then the correct answer to a query on the reference $r$ is the group of references that are coreferent to $r$, or, in other words, correspond to the same underlying entity. In our example, consider a query specified using the reference $r_{1}$ corresponding to the name 'W. Wang' in the first paper. Then the correct answer to the query is the set of references $\left\{r_{1}, r_{4}, r_{9}\right\}$. To distinguish it from the first type of entity resolution query, note that it does not include the cluster $\left\{r_{8}\right\}$ corresponding to the other entity that also has name 'W. Wang'. This second query type may be answered by first reducing it to an instance of the first type as $Q\left(R . A=r_{1} . A\right)$, and then selecting the entity corresponding to reference $r_{1}$. We denote this as $\sigma_{E(\mathcal{R})=E\left(r_{1}\right)}\left(Q\left(R \cdot A=r_{1} \cdot A\right)\right)$. In the rest of this paper, we focus only on queries of the first type.

\section{Collective Entity Resolution and Relational Clustering}

Although entity resolution for queries has not been studied in the literature, the general entity resolution problem has received a lot of attention. We review related work in detail in Section 8. In this section, we briefly review the different categories of proposed approaches before discussing how they may be adapted for query-time entity resolution.

In most entity resolution applications, data labeled with the underlying entities is hard to acquire. Our focus is on unsupervised approaches for resolving entities. Traditionally, 
attributes of individual references, such as names, affiliation, etc., for person references, are used for comparing references. A similarity measure is generally employed over attributes, and only those pairs of references that have attribute similarity above a certain threshold are considered to be co-referent. This attribute-based entity resolution approach (A) often runs into problems. In our example, it is hard to infer with just attributes that references $r_{1}$ and $r_{8}$ are not co-referent although they have the same name, while $r_{1}$ and $r_{9}$ are co-referent although their names are different.

When relations between references are available, they may also be taken into account for computing similarities in the naive relational entity resolution approach (NR) (Ananthakrishna et al., 2002; Bhattacharya \& Getoor, 2007). For computing similarities between two references, this approach additionally considers the attributes of the related references when comparing the attributes of their related references. In our example, this approach returns a higher similarity between $r_{1}$ ('W. Wang') and $r_{9}$ ('W. W. Wang') than the attribute-based approach, since they have co-authors $r_{3}$ and $r_{10}$ with very similar (identical, in this case) names. Although this approach can improve performance in some cases, it does not always work. For instance, the two 'W. Wang' references $r_{1}$ and $r_{8}$ are not co-referent, though they both have co-authors with identical names ' $\mathrm{C}$. Chen'.

Instead of considering the attribute similarities of the related references, the collective entity resolution approach (Pasula et al., 2003; Bhattacharya \& Getoor, 2004; Singla \& Domingos, 2004; McCallum \& Wellner, 2004; Li, Morie, \& Roth, 2005; Dong et al., 2005; Kalashnikov et al., 2005) takes into account the resolution decisions for them. In our previous example, the correct evidence to use for the pair of references $r_{1}$ and $r_{8}$ is that their co-author references do not map to the same entity, although they have similar names. Therefore, in order to resolve the 'W. Wang' references in the collective resolution approach, it is necessary to resolve the ' $\mathrm{C}$. Chen' references as well, instead of considering the similarity of their attributes. The collective entity resolution approach has recently been shown to improve entity resolution accuracy over the previous approaches but is computationally more challenging. The references cannot be resolved independently. Instead, any resolution decision is affected by other resolutions through hyper-edges.

In earlier work (Bhattacharya \& Getoor, 2004, 2006, 2007), we developed a relational clustering algorithm (RC-ER) for collective entity resolution using relationships. The goal of this approach is to cluster the references according to their entities taking the relationships into account. We associate a cluster label r.C with each reference to denote its current cluster membership. Starting from an initial set of clusters $\mathcal{C}=\left\{c_{i}\right\}$ of references, the algorithm iteratively merges the pair of clusters that are the most similar. To capture the collective nature of the cluster assignment, the similarity measure between pairs of clusters considers the cluster labels of the related references. The similarity of two clusters $c_{i}$ and $c_{j}$ is defined as a linear combination of their attribute similarity $\operatorname{sim}_{A}$ and their relational similarity $\operatorname{sim}_{R}$ :

$$
\operatorname{sim}\left(c_{i}, c_{j}\right)=(1-\alpha) \times \operatorname{sim}_{A}\left(c_{i}, c_{j}\right)+\alpha \times \operatorname{sim}_{R}\left(c_{i}, c_{j}\right)
$$

where $\alpha(0 \leq \alpha \leq 1)$ is the combination weight. The interesting aspect of the collective approach is the dynamic nature of the relational similarity. The similarity between two references depends on the current cluster labels of their related references, and therefore changes when related references change clusters. In our example, the similarity of the two 
clusters containing references 'W. Wang' and 'W. W. Wang' increases once their co-author references named 'A. Ansari' are assigned to the same cluster. We now briefly review how the two components of the similarity measure are defined.

Attribute Similarity: For each reference attribute, we use a similarity measure that returns a value between 0 and 1 for two attribute values indicating the degree of similarity between them. Several sophisticated similarity measures have been developed for names, and popular TF-IDF schemes may be used for other textual attributes such as keywords. The measure that works best for each attribute may be chosen. Finally, a weighted linear combination of the similarities over the different attributes yields the combined attribute similarity between two reference clusters.

Relational Similarity: Relational similarity between two clusters considers the similarity of their 'cluster neighborhoods'. The neighborhood of each cluster is defined by the hyper-edges associated with the references in that cluster. Recall that each reference $r$ is associated with one or more hyper-edges in $\mathcal{H}$. Therefore, the hyper-edge set $c . H$ for a cluster $c$ of references is defined as

$$
\text { c.H }=\bigcup_{r \in \mathcal{R} \wedge r . C=c}\{h \mid h \in \mathcal{H} \wedge r \in h . R\}
$$

This set defines the hyper-edges that connect a cluster $c$ to other clusters, and are the ones that relational similarity needs to consider. To illustrate, when all the references in our running example have been correctly clustered as in Figure 1(b), the hyper-edge set for the larger 'Wang' cluster is $\left\{h_{1}, h_{2}, h_{4}\right\}$, which are the hyper-edges associated with the references $r_{1}, r_{4}$ and $r_{9}$ in that cluster.

Given the hyper-edge set for any cluster $c$, the neighborhood $N b r(c)$ of that cluster $c$ is the set of clusters labels of the references spanned by these hyper-edges:

$$
\operatorname{Nbr}(c)=\bigcup_{h \in c . H, r \in h}\left\{c_{j} \mid c_{j}=r . C\right\}
$$

For our example 'Wang' cluster, its neighborhood consists of the 'Ansari' cluster and one of the 'Chen' clusters, which are connected by its edge-set. Then, the relational similarity measure between two clusters, considers the similarity of their cluster neighborhoods. The neighborhoods are essentially sets (or multi-sets) of cluster labels and there are many possible ways to define the similarity of two neighborhoods (Bhattacharya \& Getoor, 2007). The specific similarity measure that we use for our experiments in this paper is Jaccard similarity $^{1}$ :

$$
\operatorname{sim}_{R}\left(c_{i}, c_{j}\right)=\operatorname{Jaccard}\left(\operatorname{Nbr}\left(c_{i}\right), \operatorname{Nbr}\left(c_{j}\right)\right)
$$

Clustering Algorithm: Given the similarity measure for a pair of clusters, a greedy relational clustering algorithm can be used for collective entity resolution. Figure 2 shows high-level pseudo-code for the complete algorithm. The algorithm first identifies the candidate set of potential duplicates using a 'blocking' approach (Hernández \& Stolfo, 1995; Monge \& Elkan, 1997; McCallum, Nigam, \& Ungar, 2000). Next, it initializes the clusters

1. Jaccard similarity for two sets $A$ and $B$ is defined as $\operatorname{Jaccard}(A, B)=\frac{|A \cap B|}{|A \cup B|}$ 


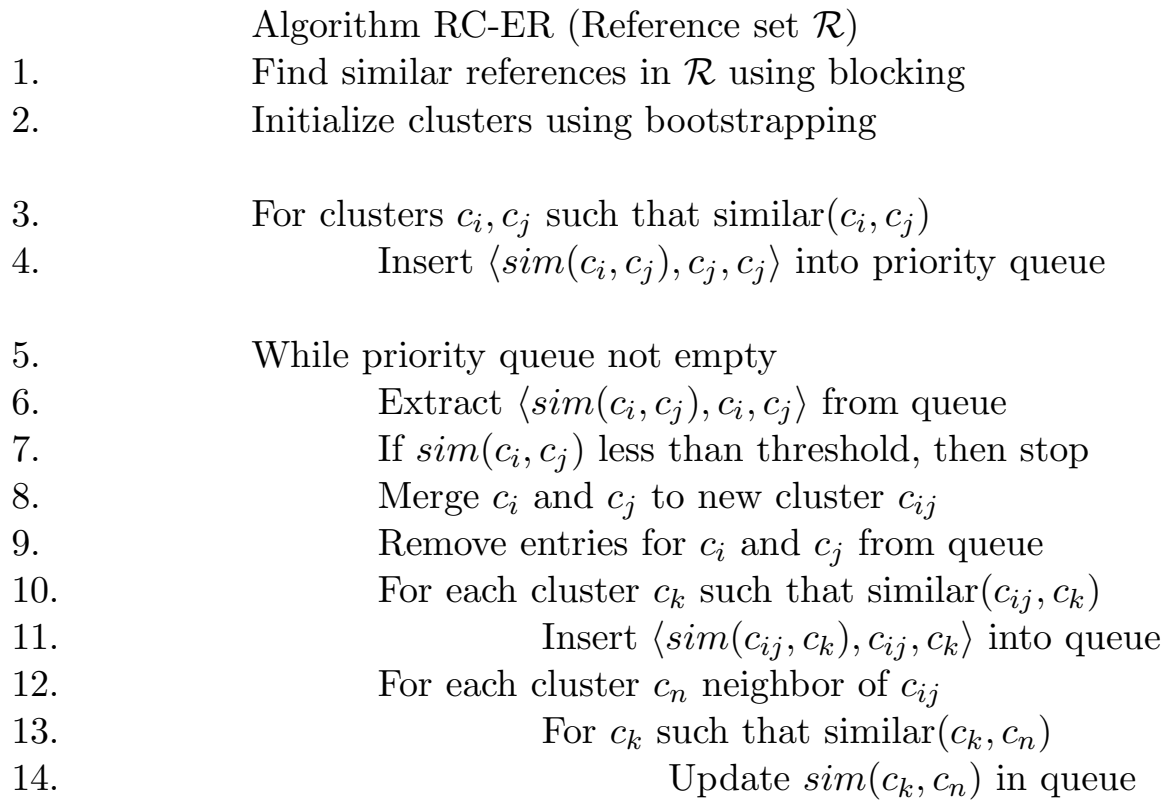

Figure 2: High-level description of the relational clustering algorithm

of references, identifies the 'similar' clusters — or potential merge-candidates - for each cluster, inserts all the merge-candidates into a priority queue and then iterates over the following steps. At each step, it identifies the current 'closest pair' of clusters from the candidate set and merges them to create a new cluster. It identifies new candidate pairs and updates the similarity measures for the 'related' cluster pairs. This is the key step where evidence flows from one resolution decision to other related ones and this distinguishes relational clustering from traditional clustering approaches. The algorithm terminates when the similarity for the closest pair falls below a threshold or when the list of potential candidates is exhausted. The algorithm is efficiently implemented to run in $O(n k \log n)$ time for $n$ references where each 'block' of similar names is connected to $k$ other blocks through the hyper-edges.

\subsection{Issues with Collective Resolution for Queries}

In previous work, we (and others) have shown that collective resolution using relationships improves entity resolution accuracy significantly for offline cleaning of databases. So, naturally, we would like to use the same approach for query-time entity resolution as well. However, while the attribute-based and naive relational approaches discussed earlier can be applied at query-time in a straight-forward fashion, that is not the case for collective resolution. Two issues come up when using collective resolution for queries. First, the set of references that influence the resolution decisions for a query need to be identified. When answering a resolution query for 'S. Russell' using the attribute-based approach, it is sufficient to consider all papers that have 'S. Russell' (or, similar names) as author name. For collective resolution, in contrast, the co-authors of these author names, such as ' $\mathrm{P}$. 
Norvig' and 'Peter Norvig', also need to be clustered according to their entities. This in turn requires clustering their co-authors and so on. So the first task is to analyze these dependencies for collective resolution and identify the references in the database that are relevant for answering a query. But this is not enough. The set of references influencing a query may be extremely large, but the query still needs to be answered quickly even though the answer may not be completely accurate. So the second issue is performing the resolution task at query-time. These are the two problems that we address in the next few sections.

\section{Analysis of Collective Resolution using Relational Clustering}

For collective entity resolution, we have seen that resolution performance for the query becomes dependent on the resolution accuracy of the related entities. Before we can analyze which other references influence entity resolution for the query and to what extent, we need to analyze the nature of this dependence for collective resolution in general. In this section, we identify the structural properties of the data that affect collective entity resolution and formally model the interdependent nature of the resolution performance. This analysis also helps us to understand when collective resolution using relational clustering helps, and, equally importantly, when it has an adverse effect as compared against traditional attribute-based resolution.

The goal of an entity resolution algorithm is to partition the set $\mathcal{R}=\left\{r_{i}\right\}$ of references into a set of clusters $\mathcal{C}=\left\{c_{i}\right\}$ according to the underlying entities $\mathcal{E}=\left\{e_{i}\right\}$. The accuracy of the resolution depends on how closely the separation of the references into clusters corresponds to the underlying entities. We consider two different measures of performance. The first measure is recall for each entity. For any entity $e_{i}$, recall counts how many pairs of references corresponding to $e_{i}$ are correctly assigned to the same computed cluster. The second measure is precision for each computed cluster. For any cluster $c_{i}$, precision counts how many pairs of references assigned to $c_{i}$ truly correspond to the same underlying entity. (Alternatively, imprecision measures how many pairs of references assigned to the cluster do not correspond to the same entity.) In the next two subsections, we analyze how these two performance metrics are influenced, first, by the attribute values of the references, and then, by the observed relationships between them.

\subsection{Influence of Attributes}

First, consider an entity resolution algorithm that follows the traditional attribute-based approach and the analysis of its performance. Such an algorithm only considers the attributes of individual references. It uses a similarity measure defined over the domain of attributes, and considers pair-wise attribute similarity between references for resolving them. Let us define two references to be $\epsilon$-similar if their attribute-similarity is at least $\epsilon$. Then, given a resolution threshold $\epsilon$, the attribute-based approach assigns a pair of references to the same cluster if and only if they are $\epsilon$-similar. To illustrate using our example, using any similarity measure defined over names and an appropriately determined similarity threshold $\epsilon$, the attribute-based approach would assign the three 'W. Wang' references $\left(r_{1}, r_{4}, r_{8}\right)$ to one cluster $c_{1}$ and the 'W. W. Wang' reference $\left(r_{9}\right)$ to a different cluster $c_{2}$. This resolution of the Wang references is not perfect in terms of precision or recall, since references $r_{1}, r_{4}$ and $r_{9}$ map to one entity $e_{1}$ and $r_{8}$ maps to a second entity $e_{2}$. Cluster $c_{1}$ has precision less 
than 1, since it incorrectly includes references for two different entities, and recall is less than 1 for entity $e_{1}$, since its references are dispersed over two different clusters.

In order to analyze the performance of this attribute-based resolution approach given an arbitrary dataset, we now characterize a dataset in terms of the attribute values of its references. Intuitively, the attribute-based approach works well when the references corresponding to the same entity are similar in terms of their attributes, and when the references corresponding to different entities are not. To capture this formally, we define two probabilities that measure the attribute-similarity of references that map to the same entity, and the attribute-similarity of those that map to different entities:

- attribute identification probability $\mathbf{a}_{\mathbf{I}}(e, \epsilon)$ : the probability that a pair of references chosen randomly from those corresponding to entity $e$ are $\epsilon$-similar to each other.

- attribute ambiguity probability $\mathbf{a}_{\mathbf{A}}\left(e_{1}, e_{2}, \epsilon\right)$ : the probability that a pair of references chosen randomly such that one corresponds to entity $e_{1}$ and the other to entity $e_{2}$ are $\epsilon$-similar to each other.

To illustrate using the four 'Wang' references, $r_{1}, r_{4}$ and $r_{9}$ correspond to the same entity $e_{1}$ and $r_{8}$ corresponds to a different entity $e_{2}$. Also, assume that for some similarity measure for names and an appropriate threshold $\epsilon$, references $r_{1}, r_{4}$ and $r_{8}$ are $\epsilon$-similar to each other. Then, of the 3 pairs of references corresponding to entity $e_{1}$, only one $\left(r_{1}\right.$ and $\left.r_{4}\right)$ is $\epsilon$-similar, so that the attribute identification probability $a_{I}\left(e_{1}, \epsilon\right)$ for entity $e_{1}$ is 0.33 . On the other hand, of the three pairs of references such that one maps to $e_{1}$ and the other to $e_{2}$, two $\left(r_{1}\right.$ and $r_{8}, r_{4}$ and $\left.r_{8}\right)$ are $\epsilon$-similar. This means that the attribute ambiguity probability $a_{A}\left(e_{1}, e_{2}, \epsilon\right)$ between $e_{1}$ and $e_{2}$ is 0.66 .

As can be seen from the above example, the performance of the attribute-based clustering algorithm can be represented in terms of these two probabilities. For any specified threshold $\epsilon$, the pairs of references for any entity that are correctly recalled are the ones that are $\epsilon$-similar, which is exactly what $a_{I}(e, \epsilon)$ captures. Therefore, the recall for any domain entity $e$ is $R(e, \epsilon)=a_{I}(e, \epsilon)$. On the other hand, consider the cluster assignment for all the references that correspond to two entities $e_{1}$ and $e_{2}$. The pairs that are incorrectly clustered together are those that correspond to two different entities, and yet are $\epsilon$-similar. This is what $a_{A}\left(e_{1}, e_{2}, \epsilon\right)$ captures. Therefore the imprecision of the cluster assignment of reference pairs corresponding to entities $e_{1}$ and $e_{2}$ is $I\left(e_{1}, e_{2}, \epsilon\right)=a_{A}\left(e_{1}, e_{2}, \epsilon\right)$. Alternatively, the precision is given by $P\left(e_{1}, e_{2}, \epsilon\right) \equiv 1-I\left(e_{1}, e_{2}, \epsilon\right)=1-a_{A}\left(e_{1}, e_{2}, \epsilon\right)$.

\subsection{Influence of Relationships}

Now, we consider the collective entity resolution approach that additionally makes use of relationships, and analyze its impact on entity resolution accuracy. Recall that we have a set $\mathcal{H}=\left\{h_{j}\right\}$ of observed co-occurrence relationships between the references. Such cooccurrences between references are useful for entity resolution when they result from strong ties or relations between their underlying entities. Specifically, we assume that references to any entity $e_{i}$ co-occur frequently with references to a small set of other entities $\left\{e_{i}^{1}, \ldots, e_{i}^{k}\right\}$, which we call the entity neighbors, denoted $N\left(e_{i}\right)$, of entity $e_{i}$. 

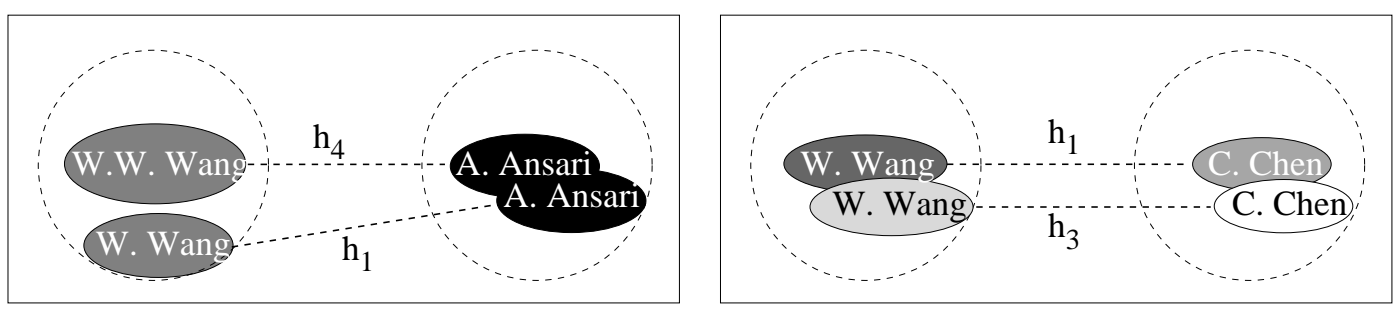

Figure 3: Illustration of (a) identifying relation and (b) ambiguous relation from running example. Dashed lines represent co-occurrence relations.

Assuming such a neighborhood relationship among the underlying entities allows us to analyze the performance of the relational clustering approach. For those reference pairs that are $\epsilon$-similar in terms of attributes, the attribute evidence is enough for resolution. But now, unlike attribute-based clustering, any pair of references that are $\delta$-similar in terms of attributes, for some $\delta<\epsilon$, are considered as candidates for being clustered together. Not all of them actually get assigned to the same cluster. For reference pairs that are in the ring of uncertainty between $\epsilon$ and $\delta$, their relationships play a role in determining if they are similar enough, and consequently, if they should be clustered together. Specifically, if references $r_{i}$ and $r_{j}$ co-occur through hyper-edge $h$ and references $r_{i}^{\prime}$ and $r_{j}^{\prime}$ co-occur through hyper-edge $h^{\prime}$, then the relational similarity of the pair $\left(r_{i}, r_{i}^{\prime}\right)$ is more when $\left(r_{j}, r_{j}^{\prime}\right)$ belong to the same cluster. In general, multiple such relationships may be needed for tipping the balance, but for simplicity, we assume for now that a single pair of related references is sufficient. In other words, $r_{i}$ and $r_{i}^{\prime}$ get assigned to the same cluster if $r_{j}$ and $r_{j}^{\prime}$ are in the same cluster.

We now analyze the impact that this approach has on entity resolution performance. Without loss of generality, assume that the $\left(r_{j}, r_{j}^{\prime}\right)$ pair get clustered together first by the relational clustering algorithm. This results in the other pair $\left(r_{i}, r_{i}^{\prime}\right)$ also getting clustered at some later iteration by considering this relational evidence. To see if this is accurate, we consider two situations, as we did with attribute evidence. The first is shown in Figure 3(a), where both pairs truly correspond to the same entity. Then the collective resolution decision is correct and we say that hyper-edges $h$ and $h^{\prime}$ are identifying relationships for that entity. Formally,

$$
\begin{aligned}
\operatorname{IRel}\left(h, h^{\prime}, e\right) \equiv & \exists r_{i}, r_{j} \in h . R, r_{i}^{\prime}, r_{j}^{\prime} \in h^{\prime} . R, \\
& E\left(r_{i}\right)=E\left(r_{i}^{\prime}\right)=e, E\left(r_{j}\right)=E\left(r_{j}^{\prime}\right)
\end{aligned}
$$

On the other hand, we may have a different scenario, in which both pairs of references correspond to two different entities. This second scenario is depicted in Figure 3(b). Then the first decision to resolve $\left(r_{j}, r_{j}^{\prime}\right)$ as co-referent is incorrect, and relational evidence obtained through hyper-edges $h$ and $h^{\prime}$ consequently leads to the incorrect resolution of $\left(r_{i}, r_{i}^{\prime}\right)$. In this situation, collective resolution hurts accuracy, and we say that $h$ and $h^{\prime}$ form ambiguous relationships for both pairs of entities, whose references may be incorrectly clustered as a result of these relationships. Formally,

$$
I A m b\left(h, h^{\prime}, e, e^{\prime}\right) \equiv \exists r_{i}, r_{j} \in h . R, r_{i}^{\prime}, r_{j}^{\prime} \in h^{\prime} . R,
$$




$$
\begin{aligned}
& E\left(r_{i}\right)=e, E\left(r_{i}^{\prime}\right)=e^{\prime}, e \neq e^{\prime}, \\
& E\left(r_{j}\right) \neq E\left(r_{j}^{\prime}\right)
\end{aligned}
$$

In general, a reference $r_{i}$ can have a co-occurrence relation $h$ that includes more than one other reference. We may think of this as multiple co-occurrence pairs involving $r_{i}$. Cluster labels of all these other references in the pairs influence resolution decisions for $r_{i}$. When resolving $r_{i}$ with another reference $r_{i}^{\prime}$ that participates in co-occurrence relation $h^{\prime}$, the fraction of common cluster labels between $h$ and $h^{\prime}$ determines whether or not $r_{i}$ and $r_{i}^{\prime}$ will be clustered together. If they are assigned to the same cluster, $h$ and $h^{\prime}$ are labeled identifying or ambiguous relationships based on whether $r_{i}$ and $r_{i}^{\prime}$ are actually co-referent or not.

Formally, we define:

- identifying relationship probability $\mathbf{r}_{\mathbf{I}}(e, \delta)$ : the probability that a randomly chosen pair of $\delta$-similar references corresponding to entity $e$ has identifying relationships $h$ and $h^{\prime}$ with some other entity.

- ambiguous relationship probability $\mathbf{r}_{\mathbf{A}}\left(e_{1}, e_{2}, \delta\right)$ : the probability that a pair of $\delta$-similar references, chosen randomly such that one corresponds to entity $e_{1}$ and the other to entity $e_{2}$, has ambiguous relationships $h$ and $h^{\prime}$ with some other pair of entities.

To illustrate these probabilities using our example, we have two 'Wang' entities, $e_{1}$ that has references $r_{1}, r_{4}$ and $r_{9}$, and $e_{2}$ that has reference $r_{8}$. Assume that the attribute threshold $\delta$ is such that all six pairs are considered potential matches. Of the three pairs of references corresponding to $e_{1}$, all of them have identifying relationships with the 'Ansari' entity. So, $r_{I}\left(e_{1}, \delta\right)=1$. To measure the relational ambiguity between the two 'Wang' entities, we consider the 3 possible pairs $\left(r_{1}\right.$ and $r_{8}, r_{4}$ and $r_{8}, r_{9}$ and $\left.r_{8}\right)$. Of these only one $\left(r_{1}\right.$ and $\left.r_{8}\right)$ pair has ambiguous relationships with two different 'Chen' entities. So, $r_{A}\left(e_{1}, e_{2}, \delta\right)=0.33$.

Given these two probabilities, we can analyze the performance of our relational clustering algorithm that combines attribute and relational evidence for collective entity resolution. It is not hard to see that the recall for any entity depends recursively on the recall of its neighbor entities. Any pair of references for entity $e$ is resolved correctly on the basis of attributes alone with probability $a_{I}(e, \epsilon)$ (the identifying attribute probability). Furthermore, it may still be resolved correctly in the presence of identifying relationships with a neighbor entity, if the related reference pair for the neighbor is resolved correctly. Denoting as $R(e, \epsilon, \delta)$ the recall for entity $e$ and that for its neighbors as $R(N(e), \epsilon, \delta)$, we have:

$$
R(e, \epsilon, \delta)=a_{I}(e, \epsilon)+\left(1-a_{I}(e, \epsilon)\right) \times r_{I}(e, \delta) \times R(N(e), \epsilon, \delta)
$$

On the other hand, consider a pair of entities $e_{1}$ and $e_{2}$. The cluster assignment for a pair of references corresponding to $e_{1}$ and $e_{2}$ is imprecise on the basis of its attributes alone with probability $a_{A}\left(e_{1}, e_{2}, \epsilon\right)$. Even otherwise, the cluster assignment can go wrong by considering relational evidence. This happens in the presence of ambiguous relationships with references corresponding to another pair of entities, if those references are also clustered 
together incorrectly. So the imprecision $I\left(e_{1}, e_{2}, \epsilon, \delta\right)$ of the cluster assignment of reference pairs corresponding to entities $e_{1}$ and $e_{2}$ turns out to be:

$$
I\left(e_{1}, e_{2}, \epsilon, \delta\right)=a_{A}\left(e_{1}, e_{2}, \epsilon\right)+\left(1-a_{A}\left(e_{1}, e_{2}, \epsilon\right)\right) \times r_{A}\left(e_{1}, e_{2}, \delta\right) \times I\left(N\left(e_{1}\right), N\left(e_{2}\right), \epsilon, \delta\right)
$$

In general, any entity $e$ has multiple neighbors $e^{i}$ in its neighborhood $N(e)$. To formalize the performance dependence on multiple neighbors, assume that if a co-occurrence involving references corresponding to $e$ is chosen at random, the probability of selecting a co-occurrence with a reference corresponding to $e^{i}$ is $p_{i}^{e}$. Then recall is given as:

$$
R(e)=a_{I}(e)+\left(1-a_{I}(e)\right) \times r_{I}(e) \times \sum_{i=1}^{|N(e)|} p_{i}^{e} R\left(e^{i}\right)
$$

Note that we have dropped $\epsilon$ and $\delta$ for notational brevity. For defining imprecision, observe that a reference corresponding to any neighbor $e_{1}^{i}$ of $e_{1}$ may co-occur with a reference for any neighbor $e_{2}^{j}$ of $e_{2}$ with probability $p_{i}^{e_{1}} p_{j}^{e_{2}}$. Then imprecision is given as:

$$
I\left(e_{1}, e_{2}\right)=a_{A}\left(e_{1}, e_{2}\right)+\left(1-a_{A}\left(e_{1}, e_{2}\right)\right) \times r_{A}\left(e_{1}, e_{2}\right) \times \sum_{i=1}^{\left|N\left(e_{1}\right)\right|} \sum_{j=1}^{\left|N\left(e_{2}\right)\right|} p_{i}^{e_{1}} p_{j}^{e_{2}} I\left(e_{1}^{i}, e_{2}^{j}\right)
$$

Given similarity thresholds $\epsilon$ and $\delta$, relational clustering increases recall beyond that achievable using attributes alone. This improvement is larger when the probability of identifying relationships is higher. On the flip side, imprecision also increases with relational clustering. Typically, a low attribute threshold $\epsilon$ that corresponds to high precision is used, and then recall is increased using relational evidence. When the probability of ambiguous relations $r_{A}$ is small, the accompanying increase in imprecision is negligible, and performance is improved overall. However, the higher the ambiguous relationship probability $r_{A}$, the less effective is relational clustering. Thus the balance between ambiguous and identifying relations determines the overall benefit of collective resolution using relational clustering. When $r_{A}$ is high compared to $r_{I}$, imprecision increases faster than recall, and overall performance is adversely affected compared to attribute-based clustering. Eq. (9) and Eq. (10) quantify this dependence of resolution performance for any entity on the nature of its relationships with other entities. In the next section, we will use these equations to design and analyze a relational clustering algorithm for answering entity resolution queries.

\section{Collective Resolution for Queries}

Our analysis of collective resolution using relational clustering showed that the resolution accuracy for any underlying entity depends on the resolution accuracy for its related/neighboring entities. For the problem of answering entity resolution queries, the goal is not to resolve all the entities in the database. We need to resolve entities for only those references that are retrieved for the query. We have seen that collective resolution leads to potential performance improvements over attribute-based resolution. We now investigate how collective resolution can be applied for answering queries to get similar improvements. The obvious hurdle is illustrated by the expressions for performance metrics in Eq. (9) and Eq. (10). They show that in order to get performance benefits for resolving the query using 
relational clustering, we need to resolve the neighboring entities as well. Furthermore, to resolve the neighboring entities, we need to resolve their neighboring entities, and so on. These other entities that need to be resolved can be very large in number, and resolving them is expensive in terms of query-processing time. Also, none of them are actually going to be retrieved as part of the answer to the query. So it is critical to identify and resolve those entities that contribute the most for improving resolution accuracy for the query. We propose a two-stage query processing strategy, consisting of an extraction phase, for identifying all the relevant references that need to be resolved for answering the query, and a resolution phase, where the relevant references that have been extracted are collectively resolved using relational clustering. Unfolding Eq. (9) and Eq. (10) starting from the query entities leads to a natural expansion process. In this section, we describe the extraction process using two novel expansion operators and, in parallel, we analyze the improvement in resolution accuracy that is obtained from considering co-occurrences.

Recall that an entity resolution query $Q(R \cdot A=a)$ is specified using an attribute $A$ and a value $a$ for it. The answer to the query consists of a partitioning of all references $r$ that have $r . A=a$ or some value $\delta$-similar to $a$. The correct answer to the query, in general, involves references from multiple entities $\left\{e_{q}\right\}$. We measure resolution accuracy for the query using two metrics as before. For each of the query entities $e_{q}$, we measure recall $R\left(e_{q}\right)$ and imprecision $I\left(e_{q}, e^{\prime}\right)$ with respect to any other entity $e^{\prime}$. Entity $e^{\prime}$ may or may not belong to $\left\{e_{q}\right\}$.

Before going into the details of our algorithm for collective resolution of queries, we briefly recall the accuracy of the attribute-based strategy of resolving a query. This approach considers all references $r$ with $r$. $A \delta$-similar to $a$, and resolves them using their attributes only. The recall that results from this approach is $R\left(e_{q}, \delta\right)=a_{I}\left(e_{q}, \delta\right)$, and the imprecision is given by $I\left(e_{q}, e^{\prime}, \delta\right)=a_{A}\left(e_{q}, e^{\prime}, \delta\right)$.

We propose two expansion operators for constructing the relevant set for an entity resolution query. We denote as level-0 references all references that are $\delta$-similar to the query attribute. These are the references that the user is interested in, and the goal is to resolve these correctly. The first operator we introduce is the attribute expansion operator $X_{A}$, or A-expansion for short. Given an attribute $A$ and a value $a$ for that attribute, $X_{A}(a, \delta)$ returns all references $r$ whose attributes $r$. $A$ exactly match $a$ or are $\delta$ similar to $a$. For a query $Q(R . A=a)$, the level- 0 references can be retrieved by expanding $Q$ as:

$$
\operatorname{Rel}^{0}(Q)=X_{A}(a, \delta)
$$

The first step in Figure 4 shows A-expansion for the query $Q(\mathcal{R}$. Name $=W . W a n g)$ in our example. It retrieves the four references $\left(r_{1}, r_{4}, r_{8}, r_{9}\right)$ that have name 'W. Wang' or 'W. W. Wang'.

To consider co-occurrence relations, we construct the level-1 references by including all references that co-occur with level- 0 references. For this, we use our second operator, which we call hyper-edge expansion $X_{H}$, or H-expansion. For any reference $r, X_{H}(r)$ returns all references that share a hyper-edge with $r$, and for a set $R$ of references $X_{H}(R)$ returns $\bigcup_{r \in R} X_{H}(r)$. Collective entity resolution requires that we consider all co-occurring references for each reference. This is achieved by performing $\mathrm{H}$-expansion on the references 


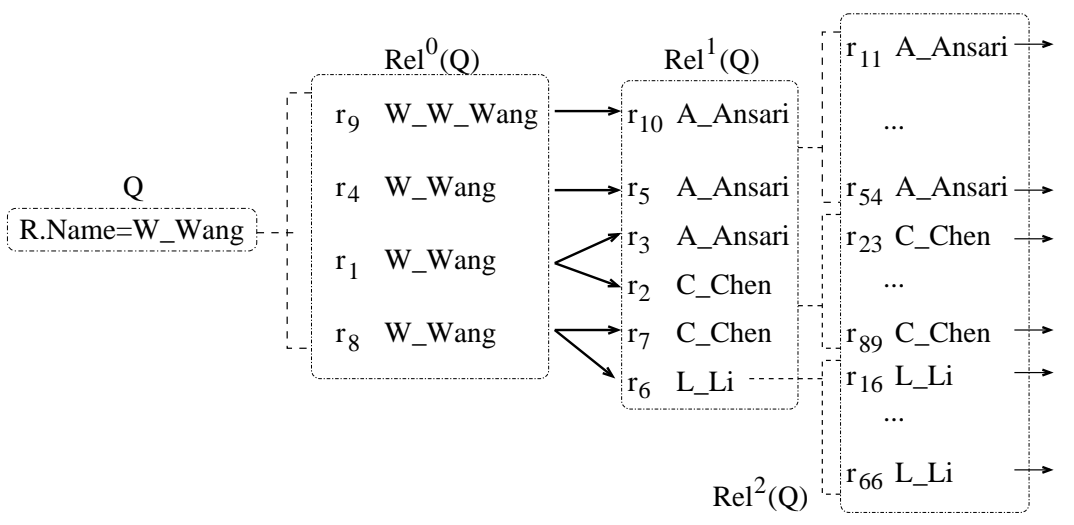

Figure 4: Relevant set for query $Q(\mathcal{R}$. Name $=$ W.Wang $)$ using H-expansion and Aexpansion alternately

at level-0 to retrieve the level-1 references:

$$
\operatorname{Rel}^{1}(Q)=X_{H}\left(\operatorname{Rel}^{0}(Q)\right)
$$

Figure 4 illustrates this operation in our example, where $X_{H}\left(r_{1}\right)$ retrieves references ' $\mathrm{C}$. Chen' $\left(r_{2}\right)$ and 'A. Ansari' $\left(r_{3}\right)$, and so on.

To perform collective resolution for the query, we additionally need to resolve the references at level-1. One option for level-1 references is attribute-based resolution using a conservative $\epsilon$-similarity to keep imprecision to a minimum. We can use our analysis technique from before to evaluate the performance for this approach. Expanding from Eq. (9), and substituting $a_{I}\left(e_{q}^{i}, \epsilon\right)$ for the recall of each neighboring entity $e_{q}^{i}$ for $e_{q}$, the recall for any query entity is:

$$
R\left(e_{q}, \epsilon, \delta\right)=a_{I}\left(e_{q}, \epsilon\right)+\left(1-a_{I}\left(e_{q}, \epsilon\right)\right) \times r_{I}\left(e_{q}, \delta\right) \times \sum_{i=1}^{k} p_{i}^{e_{q}} a_{I}\left(e_{q}^{i}, \epsilon\right)
$$

Similarly, on substituting $a_{A}\left(e_{q}^{i}, e^{j}, \epsilon\right)$ in Eq. (10) for the imprecision of each neighboring entity $e_{q}^{i}$, we get the following expression for imprecision:

$$
I\left(e_{q}, e^{\prime}, \epsilon, \delta\right)=a_{A}\left(e_{q}, e^{\prime}, \epsilon\right)+\left(1-a_{A}\left(e_{q}, e^{\prime}, \epsilon\right)\right) \times r_{A}\left(e_{q}, e^{\prime}, \delta\right) \times \sum_{i=1}^{k} \sum_{j=1}^{l} p_{i}^{e_{q}} p_{j}^{e^{\prime}} a_{A}\left(e_{q}^{i}, e^{\prime j}, \epsilon\right)
$$

To appreciate more easily the implications of considering first-order neighbors, we may assume that the attribute identification probability and the attribute ambiguity probability are the same for all the entities involved, i.e., $a_{I}(e, \epsilon)=a_{I}(\epsilon)$ and $a_{A}\left(e, e^{\prime}, \epsilon\right)=a_{A}(\epsilon)$. Then, using $\sum_{i=1}^{k} p_{i}^{e}=1$ for any entity $e$, the expression for recall simplifies to

$$
\begin{aligned}
R\left(e_{q}, \epsilon, \delta\right) & =a_{I}(\epsilon)+\left(1-a_{I}(\epsilon)\right) \times r_{I}(\delta) \times a_{I}(\epsilon) \\
& =a_{I}(\epsilon)\left[1+\left(1-a_{I}(\epsilon)\right) r_{I}(\delta)\right]
\end{aligned}
$$


Similarly, the expression for imprecision simplifies to

$$
I\left(e_{q}, e^{\prime}, \epsilon, \delta\right)=a_{A}(\epsilon)\left[1+\left(1-a_{A}(\epsilon)\right) r_{A}(\delta)\right]
$$

So we can see that attribute-clustering of the first level neighbors potentially increases recall for any query entity $e_{q}$, but imprecision goes up as well. However, when the balance between $r_{A}$ and $r_{I}$ is favorable, the increase in imprecision is insignificant and much smaller than the corresponding increase in recall, so that there is an overall performance improvement.

Can we do better than this? We can go a step further and consider co-occurrence relations for resolving the level-1 references as well. So, instead of considering attributebased resolution for references in level-1 as before, we perform collective resolution for them. We consider all of their $\delta$-similar references, which we call level-2 references $\left(\operatorname{Re}^{2}(Q)\right)$, using A-expansion:

$$
\operatorname{Rel}^{2}(Q)=X_{A}\left(\operatorname{Rel}^{1}(Q)\right)
$$

Note that we have overloaded the A-expansion operator for a set $R$ of references: $X_{A}(R)=$ $\bigcup_{r \in R} X_{A}(r . A)$. The level-3 references are the second order neighbors that co-occur with level-2 references. They are retrieved using H-expansion on the level-2 references:

$$
\operatorname{Rel}^{3}(Q)=X_{H}\left(\operatorname{Rel}^{2}(Q)\right)
$$

Finally, as with the level-1 references earlier, we resolve the level-3 references using $\epsilon$ similarity of their attributes alone.

In order to evaluate the impact on resolution accuracy for the query, we unfold the recursions in Eq. (9) and Eq. (10) up to two levels, and now substitute $a_{I}\left(e_{q}^{i}, \epsilon\right)$ for recall and $a_{A}\left(e^{i}, e^{j}, \epsilon\right)$ for imprecision for the second order neighbors. The trend in the expressions becomes clearly visible if we assume, as before, that $a_{I}$ and $a_{A}$ is identical for all entities, and, additionally, $r_{I}$ and $r_{A}$ are also the same, i.e., $r_{I}\left(e_{1}, e_{2}, \epsilon\right)=r_{I}(\epsilon)$ and $r_{A}\left(e_{1}, e_{2}, \delta\right)=r_{A}(\delta)$. Then, we can work through a few algebraic steps to get the following expressions for recall and precision for any query entity $e_{q}$ :

$$
\begin{aligned}
R\left(e_{q}\right) & =a_{I}\left[1+\left(1-a_{I}\right) r_{I}+\left(1-a_{I}\right)^{2} r_{I}^{2}\right] \\
I\left(e_{q}, e^{\prime}\right) & =a_{A}\left[1+\left(1-a_{A}\right) r_{A}+\left(1-a_{A}\right)^{2} r_{A}^{2}\right]
\end{aligned}
$$

We can continue to unfold the recursion further and grow the relevant set for the query. Formally, the expansion process alternates between A-expansion and H-expansion:

$$
\begin{aligned}
& \operatorname{Rel}^{i}(Q)=X_{A}(Q) \quad \text { for } i=0 \\
& X_{H}\left(\operatorname{Rel}^{i-1}(Q)\right) \quad \text { for odd } i \\
& X_{A}\left(\operatorname{Rel}^{i-1}(Q)\right) \quad \text { for even } i
\end{aligned}
$$

As we proceed recursively and consider higher order co-occurrences for the query, additional terms appear in the expressions for precision and recall. But this does not imply that we need to continue this process to arbitrary levels to get optimum benefit. Using our simplifying assumptions about the attribute and relational probabilities, the expressions for both recall and imprecision for $n^{\text {th }}$ order co-occurrences turns out to be geometric 
progressions with $n+1$ terms. The common ratio for the two geometric progressions are $\left(1-a_{I}(\epsilon)\right) r_{I}(\delta)$ and $\left(1-a_{A}(\epsilon)\right) r_{A}(\delta)$ respectively. Typically, both of these ratios are significantly smaller than 1 , and therefore converge very quickly with increasing co-occurrence level. So the improvement in resolution accuracy for the query $Q$ falls off quickly with expansion depth, and we can terminate the expansion process at some cut-off depth $d^{*}$ without compromising on accuracy:

$$
\operatorname{Rel}(Q)=\bigcup_{i=0}^{d^{*}} \operatorname{Rel}^{i}(Q)
$$

Of course, the assumptions about the attribute and relational probabilities being entityindependent do not hold in practice, so that the performance trends for increasing levels of co-occurrence cannot be exactly captured by geometric progressions with a common ratio for successive terms. But the converging trends for both of them still hold in general, and the rate of convergence is still determined by the four probabilities $a_{I}, a_{A}, r_{I}$ and $r_{A}$ for the entities that are encountered during the expansion process. Intuitively, smaller values for $r_{I}$ and $r_{A}$ indicate less sensitivity to co-occurrences, and the convergence is quicker. On the other hand, higher values of $a_{I}$ and $a_{A}$ mean that more entities are resolved based on attributes alone - correctly or incorrectly - and the impact of co-occurrence relations is smaller. Therefore convergence is quicker for higher values of $a_{I}$ and $a_{A}$.

Apart from imposing a cutoff on the expansion depth, the size of the relevant set can also be significantly reduced by restricting attribute expansion beyond level- 0 to exact A-expansion $X_{A}^{e}(r)$. This only considers references with exactly the same attribute as $r$ and disregards other $\delta$-similar references. Interestingly, we can show that the restricted strategy that alternates between exact A-expansion and $\mathrm{H}$-expansion does not reduce recall significantly.

\section{Adaptive Query Expansion}

The limited depth query expansion strategy proposed in the previous section is an effective approach that is able to answer queries quickly and accurately for many domains. However, for some domains, the size of the relevant set that is generated can be extremely large even for small expansion depths, and as a result, the retrieved references cannot be resolved at query-time. In this section, we propose adaptive strategies based on estimating the 'ambiguity' of individual references that makes our algorithm even more efficient while preserving accuracy.

The main reason behind this explosive growth of the relevant set with increasing levels is that our query expansion strategy from the previous section is unconstrained - it treats all co-occurrences as equally important for resolving any entity. It blindly expands all references in the current relevant set, and also includes all new references generated by an expansion operation. Given the limited time to process a query, this approach is infeasible for domains that have dense relationships. Our solution is to identify the references that are likely to be the most helpful for resolving the query, and to focus on only those references. To illustrate using our example from Figure 4, observe that 'Chen' and 'Li' are significantly more common or 'ambiguous' names than 'Ansari' - even different 'W. Wang' entities are likely to have collaborators named 'Chen' or 'Li'. Therefore, when h-expanding $\operatorname{Rel}^{0}\left(r_{q}\right)$ for 
'W. Wang', 'Ansari' is more informative than 'Chen' or 'Li'. Similarly, when n-expanding $\operatorname{Rel}^{1}\left(r_{q}\right)$, we can choose not to expand the name 'A. Ansari' any further, since two 'A. Ansari' references are very likely to be coreferent. But we need more evidence for the 'Chen's and the 'Li's.

To describe this formally, the ambiguity of a value $a$ for an attribute $A$ is the probability that any two references $r_{i}$ and $r_{j}$ in the database that have $r_{i} . A=r_{j} . A=a$ are not coreferent: $A m b(a)=P\left(E\left(r_{i}\right) \neq E\left(r_{j}\right) \mid r_{i} \cdot A=r_{j} . A=a\right)$. The goal of adaptive expansion is to add less ambiguous references to the relevant set and to expand the most ambiguous references currently in the relevant set. We first define adaptive versions of our two expansion operators treating the ambiguity estimation process as a black-box, and then look at ways to estimate ambiguity of references.

\subsection{Adaptive Expansion Operators}

The goal of adaptive expansion is to selectively choose the references to expand from the current relevant set, and also the new references that are included at every expansion step. For adaptive hyper-edge expansion, we set an upper-bound $h_{\max }$ on the number of new references that h-expansion at a particular level can generate. Formally, we want $\left|X_{H}\left(\operatorname{Rel}^{i}(Q)\right)\right| \leq h_{\max }\left|\operatorname{Rel}^{i}(Q)\right|$. The value of $h_{\max }$ may depend on depth $i$ but should be small enough to rule out full h-expansion of the current relevant set. Then, given $h_{\max }$, our strategy is to choose the least ambiguous references from $X_{H}\left(\operatorname{Rel}^{i}(Q)\right)$, since they provide the most informative evidence for resolving the references in $\operatorname{Rel}^{i}(Q)$. To achieve this, we sort the h-expanded references in increasing order of ambiguity and select the first $k$ from them, where $k=h_{\text {max }}\left|\operatorname{Rel}^{i}(Q)\right|$.

$$
\operatorname{Rel}_{\text {adapt }}^{i}\left(Q, h_{\text {max }}\right)=\operatorname{Least} A m b\left(k, X_{H}\left(\operatorname{Rel}_{\text {adapt }}^{i-1}(Q)\right)\right)
$$

The setting for adaptive attribute expansion is very similar. For some positive number $a_{\max }$, exact a-expansion of $\operatorname{Re}^{i}(Q)$ is allowed to include at most $a_{\max }\left|\operatorname{Re}^{i}(Q)\right|$ references. Note that now the selection preference needs to be flipped - more ambiguous names need more evidence, so they are expanded first. So we can sort $X_{A}^{e}\left(\operatorname{Rel}^{i}(Q)\right)$ in decreasing order of ambiguity and select the first $k$ from the sorted list, where $k=a_{\text {max }}\left|\operatorname{Rel}^{i}(Q)\right|$. But this could potentially retrieve only references for the most ambiguous name, totally ignoring references with any other name. To avoid this, we choose the top $k$ ambiguous references from $\operatorname{Rel}^{i}(Q)$ before expansion, and then expand the references so chosen.

$$
\operatorname{Re}_{\text {adapt }}^{i}\left(Q, n_{\text {max }}\right)=X_{A}^{e}\left(\operatorname{Most} A m b\left(k, \operatorname{Rel}_{\text {adapt }}^{i}(Q)\right)\right)
$$

Though this cannot directly control the number of new references added, $\mu_{r} \times k$ is a reasonable estimate, where $\mu_{r}$ is the average number of references per name.

\subsection{Ambiguity Estimation}

The adaptive expansion scheme proposed in this section is crucially dependent on the estimates of name ambiguity. We now describe one possible scheme that worked quite well. Recall that we want to estimate the probability that two randomly picked references with value $a$ for attribute $A$ correspond to different entities. For a reference attribute $A_{1}$, denoted 


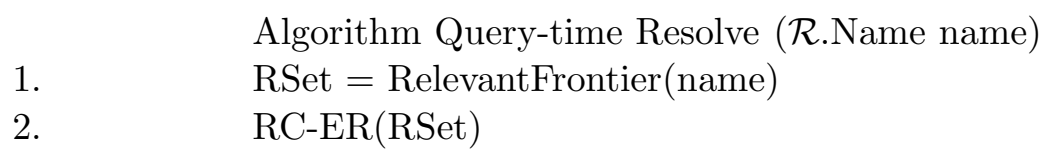

$\begin{array}{ll} & \text { Algorithm FindRelevantRefs }(\mathcal{R} \text {.Name name }) \\ 1 . & \text { Initialize RSet to }\{\} \\ 5 . & \text { Initialize depth to } 0 \\ 3 . & \text { Initialize FrontierRefs to }\{\} \\ 4 . & \text { While depth }<\mathrm{d}^{*} \\ 5 . & \text { If depth is even or } 0 \\ 6 . & \mathrm{R}=X_{A}(\text { FrontierRefs }) \\ 7 . & \left.\mathrm{R}=X_{H} \text { (FrontierRefs }\right) \\ 8 . & \text { FrontierRefs }=\mathrm{R} \\ 9 . & \text { Add FrontierRefs to RSet } \\ 10 . & \text { Increment depth } \\ 10 . & \text { Return RSet }\end{array}$

Figure 5: High-level description of the query-time entity resolution algorithm

$R . A_{1}$, a naive estimate for the ambiguity of a value of $n$ for the attribute is:

$$
A m b\left(r . A_{1}\right)=\frac{\left|\sigma_{R \cdot A_{1}=r \cdot A_{1}}(R)\right|}{|R|}
$$

where $\left|\sigma_{R . A_{1}=r . A_{1}}(R)\right|$ denotes the number of references with value $r . A_{1}$ for $A_{1}$. This estimate is clearly not good since the number of references with a certain attribute value does not always match the number of different entity labels for that attribute. We can do much better if we have an additional attribute $A_{2}$. Given $A_{2}$, the ambiguity for value of $A_{1}$ can be estimated as

$$
A m b\left(r . A_{1} \mid r \cdot A_{2}\right)=\frac{\left|\delta\left(\pi_{R \cdot A_{2}}\left(\sigma_{R \cdot A_{1}=r \cdot A_{1}}(R)\right)\right)\right|}{|R|},
$$

where $\left|\delta\left(\pi_{R . A_{2}}\left(\sigma_{R . A_{1}=r . A_{1}}(R)\right)\right)\right|$ is the number of distinct values observed for $A_{2}$ in references with $R . A_{1}=r \cdot A_{1}$. For example, we can estimate the ambiguity of a last name by counting the number of different first names observed for it. This provides a better estimate of the ambiguity of any value of an attribute $A_{1}$, when $A_{2}$ is not correlated with $A_{1}$. When multiple such uncorrelated attributes $A_{i}$ are available for references, this approach can be generalized to obtain better ambiguity estimates.

Putting everything together, high-level pseudo code for the query-time entity resolution algorithm is shown in Figure 5. The algorithm works in two stages - first, it identifies the relevant set of references given an entity name as a query, and then it performs relational clustering on the extracted relevant references. The relevant references are extracted using the recursive process that we have already seen. The relevant references at any depth $i$ are obtained by expanding the relevant references at depth $i-1$, the expansion being dependent 
of whether it is an odd step or an even step. The actual expansion operator that is used may either be unconstrained or adaptive.

\section{Empirical Evaluation}

For experimental evaluation of our query-time resolution strategies, we used both realworld and synthetically generated datasets. First, we describe our real datasets and the experiments performed on them and then we move on to our experiments on synthetic data.

\subsection{Experiments on Real Data}

For real-world data, we used two citation datasets with very different characteristics. The first dataset, arXiv, contains papers from high energy physics and was used in KDD Cup $2003^{2}$. It has 58,515 references to 9,200 authors, contained in 29,555 publications. The number of author references per publication ranges from 1 to 10 with an average of 1.90. Our second dataset is the Elsevier BioBase database ${ }^{3}$ of publications from biology used in the recent IBM KDD-Challenge competition. It includes all publications under 'Immunology and Infectious Diseases' between years 1998 and 2001. This dataset contains 156,156 publications with 831,991 author references. The number of author references per publication is significantly higher than arXiv and ranges from 1 to 100 (average 5.3). All names in this database only have initials for first and middle names (if available), unlike arXiv, which has both initialed and complete names. The number of distinct names in BioBase is 303,693, with the number of references for any name ranging from 1 to 193 (average 2.7). Unlike arXiv, BioBase includes keywords, topic classification, language, country of correspondence and affiliation of the corresponding author as attributes of each paper, all of which we use as attributes for resolution in addition to author names. BioBase is diverse in terms of these attributes, covering 20 languages, 136 countries, 1,282 topic classifications and 7,798 keywords.

For entity resolution queries in arXiv, we selected all ambiguous names that correspond to more than one author entity. This gave us 75 queries, with the number of true entities for the selected names varying from 2 to 11 (average 2.4). For BioBase, we selected as queries the top 100 author names with the highest number of references. The average number of references for each of these 100 names is 106, and the number of entities for the selected names ranges from 1 to 100 (average 32), thereby providing a wide variety of entity resolution settings over the queries.

\subsubsection{Relevant Set Size Vs. Resolution Time}

We begin by exploring the growth rate of the relevant set for a query over expansion depth in the two datasets. Figure 6(a) plots the size of the relevant set for a sample query on the name 'T. Lee' for arXiv and 'M. Yamashita' for BioBase. The growth rate for the arXiv query is moderate. The number of references with name ' $\mathrm{T}$. Lee' is 7 , which is the number of relevant references at depth 0 , and the size grows to 7,500 by depth 7 . In contrast, for BioBase the plots clearly demonstrate the exponential growth of the relevant references

2. http://www.cs.cornell.edu/projects/kddcup/index.html

3. http://help.sciencedirect.com/robo/projects/sdhelp/about_biobase.htm 

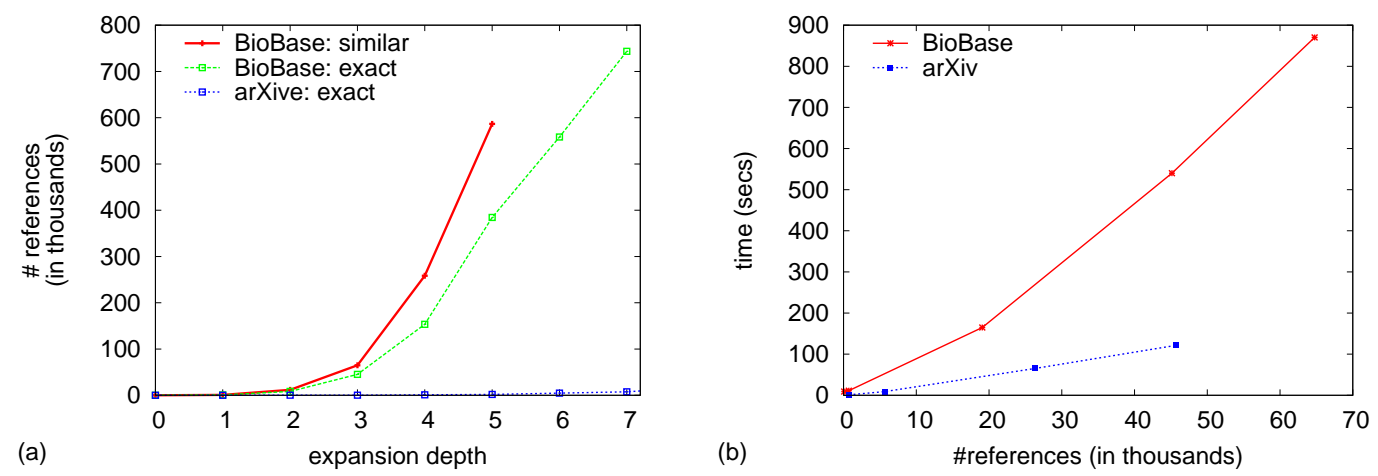

Figure 6: (a) Size of the relevant set for increasing expansion depth for sample queries in arXiv and BioBase (b) Execution time of RC-ER with increasing number of references

with depth for both name expansion strategies. There are 84 relevant references at depth 0 . When references are expanded using name similarity expansion, there are 722 relevant references at depth 1, 65,000 at depth 3 and more than 586,000 at depth 5 . This is for a very restricted similarity measure where two names are considered similar only if their first initials match, and the last names have the same first character and differ overall by at most 2 characters. A more liberal measure would result in a significantly faster growth. We also observe that for exact expansion, the growth is slower but we still have 45,000 references at depth $3,384,000$ at depth 5 and 783,000 by depth 7 . It is interesting to note that the growth slows down beyond depth 5; but this is because most of the references in the entire dataset are already covered at that depth (BioBase has 831,991 references in total). The growth rates for these two examples from arXiv and BioBase are typical for all of our queries in these two datasets.

Next, in Figure 6(b), we observe how the relational clustering algorithm RC-ER scales with increasing number of references in the relevant set. All execution times are reported on a Dell Precision 870 server with $3.2 \mathrm{GHz}$ Intel Xeon processor and $3 \mathrm{~GB}$ of memory. The plot shows that the algorithm scales well with increasing references, but the gradient is different for the two datasets. This is mainly due to the difference in the average number of references per hyper-edge. This suggests that for arXiv, RC-ER is capable of handling the relevant sets generated using unconstrained expansion. But for BioBase, it would require up to 600 secs for 40,000 references, and up to 900 secs for 65,000 . So it is clearly not possible to use RC-ER with unconstrained expansion for query-time resolution in BioBase even for depth 3.

\subsubsection{Entity Resolution Accuracy for Queries}

In our next experiment, we evaluate several algorithms for entity resolution queries. We compare entity resolution accuracy of the pair-wise co-reference decisions using the F1 measure (which is the harmonic mean of precision and recall). For a fair comparison, we consider the best F1 for each of these algorithms over all possible thresholds for determining 
Table 1: Average entity resolution accuracy (F1) for different algorithms over 75 arXiv queries and 100 BioBase queries

\begin{tabular}{|l|c|c|}
\hline & arXiv & BioBase \\
\hline \hline A & 0.721 & 0.701 \\
A* & 0.778 & 0.687 \\
NR & 0.956 & 0.710 \\
NR* & 0.952 & 0.753 \\
RC-ER Depth-1 & 0.964 & 0.813 \\
RC-ER Depth-3 & 0.970 & 0.820 \\
\hline
\end{tabular}

duplicates. For the algorithms, we compare attribute-based entity resolution (A), naive relational entity resolution (NR) that uses attributes of related references, and our relational clustering algorithm for collective entity resolution (RC-ER) using unconstrained expansion up to depth 3 . We also consider transitive closures over the pair-wise decisions for the first two approaches ( $\mathbf{A}^{*}$ and $\mathbf{N R}^{*}$ ). For attribute similarity, we use the Soft TF-IDF with Jaro-Winkler similarity for names, which has been shown to perform the best for namebased resolution (Bilenko, Mooney, Cohen, Ravikumar, \& Fienberg, 2003), and TF-IDF similarity for the other textual attributes.

The average F1 scores over all queries are shown in Table 1 for each algorithm in the two datasets. It shows that RC-ER improves accuracy significantly over the baselines. For example in BioBase, the improvement is $21 \%$ over $\mathbf{A}$ and $\mathbf{N R}, 25 \%$ over $\mathbf{A}^{*}$ and $13 \%$ over NR*. This demonstrates the potential benefits of collective resolution for answering queries, and validates recent results in the context of offline entity resolution (Bhattacharya \& Getoor, 2004, 2007; Singla \& Domingos, 2004; Dong et al., 2005; McCallum \& Wellner, 2004). In our earlier work (Bhattacharya \& Getoor, 2007) we have demonstrated using extensive experiments on real and synthetic datasets how our relational clustering algorithm (RC-ER) improves entity resolution performance over traditional baselines in the context of offline data cleaning, where the entire database is cleaned as a whole. The numbers in Table 1 confirm that similar improvements can be obtained for localized resolution as well. As predicted by our analysis, most of the accuracy improvement comes from the depth-1 relevant references. For 56 out of the 100 BioBase queries, accuracy does not improve beyond the depth- 1 relevant references. For the remaining 44 queries, the average improvement is $2 \%$. However, for 8 of the most ambiguous queries, accuracy improves by more than $5 \%$, the biggest improvement being as high as $27 \%$ (from 0.67 to $0.85 \mathrm{~F} 1$ ). Such instances are fewer for arXiv, but the biggest improvement is $37.5 \%$ (from 0.727 to 1.0). On one hand, this shows that considering related records and resolving them collectively leads to significant improvement in accuracy. On the other hand, it also demonstrates that while there are potential benefits to considering higher order neighbors, they fall off quickly beyond depth 1 . This also serves to validate our analysis of collective query resolution in Section 4. 
Table 2: Average query processing time with unconstrained expansion

\begin{tabular}{|l|c|c|}
\hline & arXiv & BioBase \\
\hline \hline A & 0.41 & 9.35 \\
A* & 0.41 & 9.59 \\
NR & 0.43 & 28.54 \\
NR* & 0.428 & 28.69 \\
RC-ER Depth-1 & 0.45 & 11.88 \\
RC-ER Depth-3 & 1.36 & 606.98 \\
\hline
\end{tabular}

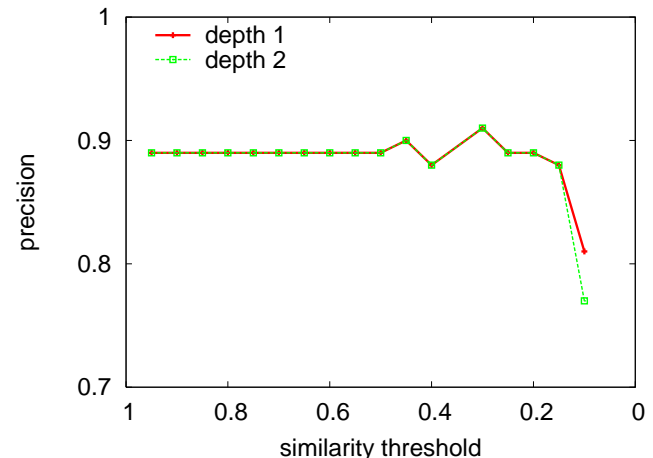

(a)

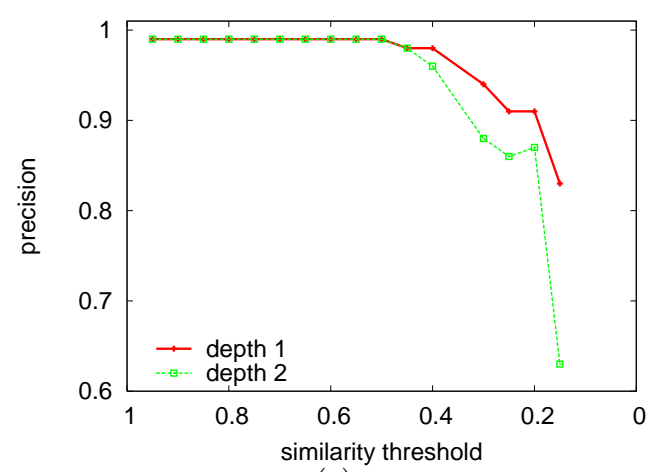

(c)

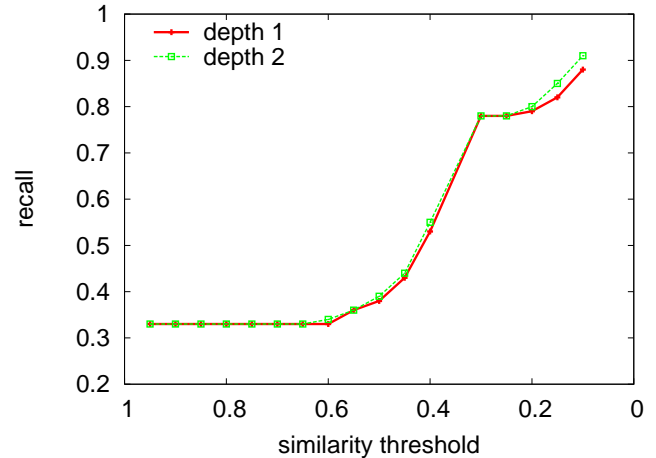

(b)

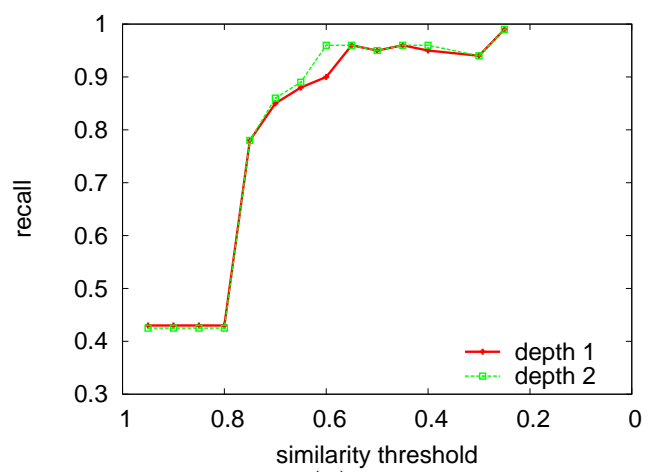

(d)

Figure 7: Average precision and recall at different similarity thresholds for (a-b) BioBase and (c-d) arXiv

The last two rows of Table 1 show the converging nature of entity resolution performance with increasing depth. We verify this explicitly for precision and recall in Figure 7 . The top two plots show average precision and recall over BioBase queries at different similarity thresholds for RC-ER. The bottom two plots show the same for arXiv. We can see that the precision curve at depth 1 coincides with or stays marginally above the precision curve at depth 3 for both BioBase and arXiv. The recall curves show the opposite trend - recall 
marginally improves for depth 3. This is in agreement with our derived expressions for precision and recall for increasing depth in Eq. (12). The difference in recall between depths 1 and 3 can be quantified as $a_{I}\left(1-a_{I}\right)^{2} r_{I}^{2}$, and the difference in precision as $a_{A}\left(1-a_{A}\right)^{2} r_{A}^{2}$. The explanation for the small difference between average precision and recall in these two plots is that both of these factors, when averaged over all queries, are significantly smaller than 1 for arXiv and BioBase. We will investigate this converging nature of performance in more detail by varying these structural properties in our experiments with synthetic data in Section 7.2.

\subsubsection{Reducing Time With Adaptive Expansion}

The first set of experiments show the effectiveness of our two-phase query processing strategy in terms of entity resolution performance. The challenge, as we have described earlier, is in obtaining these benefits in real-time. So, next, we focus on the time that is required to process these queries in the two datasets using unconstrained expansion up to depth 3 . The results are shown in Table 2. For arXiv, the average processing time for depth-3 expansion is 1.36 secs, with 406 relevant references on average. This shows that our two-phase strategy with unconstrained expansion is a practical processing strategy for entity resolution queries - it resolves the query entities accurately, and extremely quickly as well. However, for BioBase, the average number of references reached by depth 3 is more that 44,000, and the time taken to resolve them collectively is more than 10 minutes. This is unacceptable for answering queries, and next we focus on how the processing time is improved using our proposed adaptive strategies. Note that the time taken for depth-1 expansion is around 12 secs, which is close to that for the attribute-based baseline $(\mathbf{A})$ and less than the time for the naive relational algorithm (NR).

Since unconstrained expansion is effective for arXiv, we focus only on BioBase for evaluating our adaptive strategies. For estimating ambiguity of references, we use last names with first initial as the secondary attribute. This results in very good estimates of ambiguity — the ambiguity estimate for a name is strongly correlated (correlation coeff. 0.8) with the number of entities for that name. First, we evaluate adaptive H-expansion. Since H-expansion occurs first at depth 1, for each query, we construct the relevant set with cutoff depth $d^{*}=1$, and use adaptive H-expansion for depth 1 . The expansion upper-bound $h_{\max }$ is set to 4 . We compare three different adaptive H-expansion strategies: (a) choosing the least ambiguous references, (b) choosing the most ambiguous references and (c) random selection. Then, for each query, we evaluate entity resolution accuracy using RC-ER on the relevant sets constructed using these three adaptive strategies. The average accuracies for the three strategies over all 100 queries are shown in the first column of Table 3. Least ambiguous selection, which is the strategy that we propose, clearly shows the biggest improvement and most ambiguous the smallest, while random selection is in between. Notably, even without many of the depth-1 references, all of them improve accuracy over $\mathbf{N R}^{*}$ by virtue of collective resolution.

We perform a similar set of experiments for evaluating adaptive attribute expansion. Recall that depth 2 is the lowest depth where adaptive attribute expansion is performed. So for each query, we construct the relevant set with $d^{*}=3$ using adaptive A-expansion at depth 1 and unconstrained H-expansion at depths 1 and 3. The expansion upper-bound 
Table 3: Avg. resolution accuracy in F1 with different adaptive expansion strategies

\begin{tabular}{|l|cc|}
\hline & H-expansion & A-expansion \\
\hline \hline Least Ambiguous & 0.790 & 0.815 \\
Most Ambiguous & 0.761 & 0.821 \\
Random & 0.770 & 0.820 \\
\hline
\end{tabular}

$a_{\max }$ is set to 0.2 , so that on average 1 out of 5 names are expanded. Again, we compare three strategies: (a) expanding the least ambiguous names, (b) expanding the most ambiguous names and (c) random expansion. The average accuracies for the three schemes over all 100 queries are listed in the second column of Table 3. The experiment with adaptive Aexpansion does not bring out the difference between the three schemes as clearly as adaptive H-expansion. This is because we are comparing A-expansion at depth 2 and, on average, not much improvement can be obtained beyond depth 1 because of a ceiling effect. But it shows that almost all the benefit up to depth 3 comes from our proposed strategy of expanding the most ambiguous names.

The above two experiments demonstrate the effectiveness of the two adaptive expansion schemes in isolation. Now, we look at the results when we use them together. For each of the 100 queries, we construct the relevant set $\operatorname{Rel}\left(r_{q}\right)$ with $d^{*}=3$ using adaptive Hexpansion and adaptive exact A-expansion. Since most of the improvement from collective resolution comes from depth-1 references, we consider two different experiments. In the first experiment (AX-2), we use adaptive expansion only at depths 2 and beyond, and unconstrained H-expansion at depth 1. In the second experiment (AX-1), we use adaptive H-expansion even at depth 1 , with $h_{\max }=6$. For both of them, we use adaptive expansion at higher depths 2 and 3 with parameters $h_{\max }=3$ at 3 and $a_{\max }=0.2$ at 2 .

Table 4: Comparison between unconstrained and adaptive expansion for BioBase

\begin{tabular}{|l|ccc|}
\hline & Unconstrained & AX-2 & AX-1 \\
\hline \hline relevant-set size & $44,129.5$ & $5,510.52$ & $3,743.52$ \\
time (cpu secs) & 606.98 & 43.44 & 31.28 \\
accuracy (F1) & 0.821 & 0.818 & 0.820 \\
\hline
\end{tabular}

In Table 4, we compare the two adaptive schemes against unconstrained expansion with $d^{*}=3$ over all queries. Clearly, accuracy remains almost unaffected for both schemes. First, we note that AX-2 matches the accuracy of unconstrained expansion, and shows almost the same improvement over depth 1 . This accuracy is achieved even though it uses adaptive expansion that expands a small fraction of $\operatorname{Re}^{1}(Q)$, and thereby reduces the average size of the relevant set from 44,000 to 5,500. More significantly, AX-1 also matches this improvement even without including many depth-1 references. This reduction in the size of the relevant set has an immense impact on the query processing time. The average processing time drops from more than 600 secs for unconstrained expansion to 43 secs for 

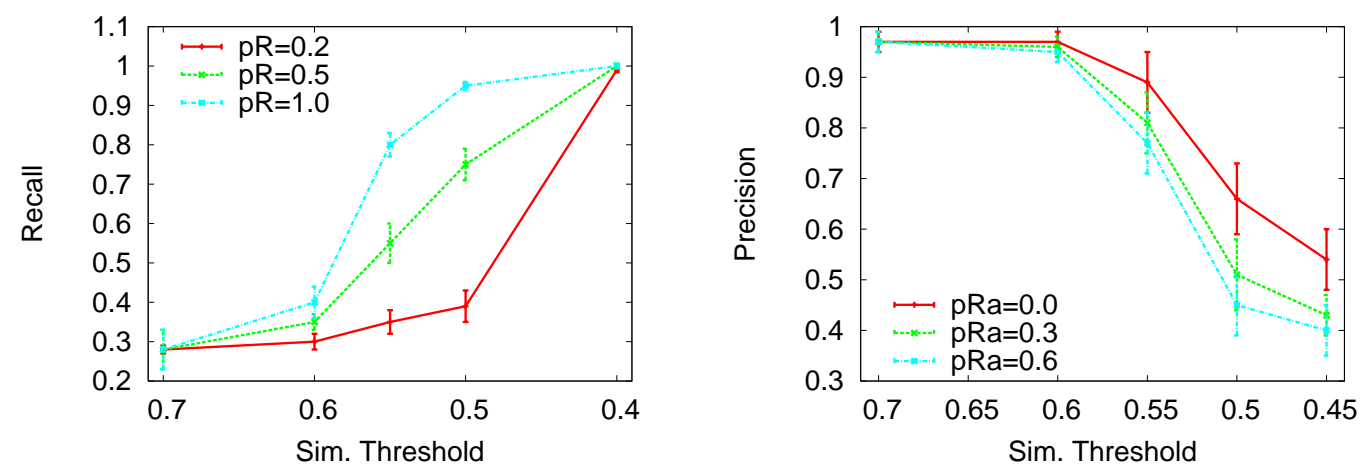

Figure 8: Effect of (a) identifying relations on recall and (b) ambiguous relations on precision for collective clustering. Error bars show standard deviation.

AX-2, and further to just 31 secs for AX-1, thus making it possible to use collective entity resolution for query-time resolution.

\subsubsection{Adaptive Depth Selection}

As a further improvement, we investigate if processing time can be reduced by setting the expansion depth $d^{*}$ adaptively, depending on the ambiguity of the query name, as compared to a fixed $d^{*}$ for all queries. In a simple setup, we set $d^{*}$ to 1 for queries where the number of different first initials for a last name is less than 10 (out of 26), and explore depth 2 only for more ambiguous queries. This reduces expansion depth from 2 to 1 for 18 out of the 100 queries. As a result, the average processing time for these queries is reduced by $35 \%$ to 11.5 secs from 17.7 secs with no reduction in accuracy. For three of these queries, the original processing time at depth 2 is greater than 30 secs. In these preliminary experiments, we only evaluated our original set of 100 queries that are inherently ambiguous. In a more general setting, where a bigger fraction of queries have lower ambiguity, the impact is expected to be even more significant.

\subsection{Experiments using Synthetic Data}

In addition to experiments on real datasets, we performed experiments on synthetically generated data. This enables us to reason beyond specific datasets, and also to empirically verify our performance analysis for relational clustering in general, and more specifically for entity resolution queries. We have designed a generator for synthetic data (Bhattacharya \& Getoor, 2007) that allows us to control different properties of the underlying entities and the relations between them, and also of the observed co-occurrence relationships between the entity references. Among other properties, we can control the number of entities, the average number of neighbor entities per entity, and the number and average size of observed co-occurrences. Additionally, we can control the ambiguity of entity attributes, and the number of ambiguous relationships between entities. We present an overview of the synthetic data generation process in Appendix A. 

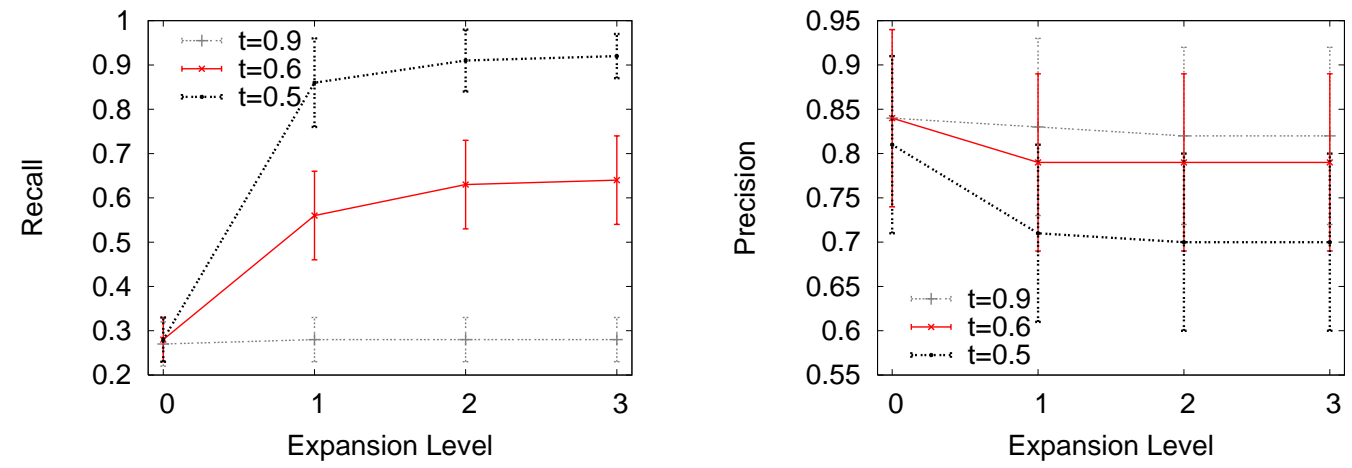

Figure 9: Change in (a) precision and (b) recall for increasing expansion levels used for collective clustering. Error bars show standard deviation.

We have performed a number of different experiments on synthetic data. In the first set of experiments, we investigate the influence of identifying relationships on collective resolution using relational clustering. We generate 500 co-occurrence relations from the same 100 entities and 200 entity-entity relationships, using varying probability of co-occurrences $p^{R}=\{0.2,0.5,1.0\}$ in the data. The probability of ambiguous relationships is held fixed, so that higher $p^{R}$ translates to higher probability of identifying co-occurrences in the data. Figure 8(a) shows recall at different similarity thresholds for three different co-occurrence probabilities. The results confirm that recall increases progressively with more identifying relationships at all thresholds. The curves for $p^{R}=0.5$ and $p^{R}=1.0$ flatten out only when no further recall is achievable.

Next, we observe the effect of ambiguous relations on the precision of collective resolution using relational clustering. We add 200 binary relationships between 100 entities in three stages with increasing ambiguous relationship probability $\left(p_{a}^{R}=\{0,0.3,0.6\}\right)$. Then we perform collective resolution on 500 co-occurrence relations generated from each of these three settings. In Figure 8(b) we plot precision at different similarity threshold for three different values of $p_{a}^{R}$. The plots confirm the progressive decrease in precision for all thresholds with higher $p_{a}^{R}$. For both experiments, the results are averaged over 200 different runs.

Next, we evaluate collective resolution for queries. Recall that the last two rows in Table 1 clearly demonstrate the converging nature of performance over increasing expansion levels for queries on real datasets. We ran further experiments on synthetic data to verify this trend. In each run, we generated 2,500 co-occurrence relations from 500 entities having an average of 2 neighbors per entity. Then we performed localized collective clustering in each case, using as query the most ambiguous attribute value (that corresponds to the highest number of underlying entities). In Figure 9(c) and (d), we show how recall and precision change with increasing expansion level for a query. Recall improves with increasing expansion level, while precision decreases overall, as is predicted by our analysis. Importantly, recall increases at a significantly faster rate than that for the decrease in precision. In general, the rate of increase/decrease depends on the structural properties of the data, as we have shown in our analysis. In other experiments, we have seen different rates of 
change, but the overall trend remains the same. Our analysis also showed that precision and recall converge quickly over increasing expansion levels. This too is confirmed by the two plots where the curves flatten out by level 3 .

\subsection{Current Limitations}

Finally, we discuss two of the current limitations of our collective entity resolution approach. Recall that the similarity measure in Eqn. 1 involves a weighting parameter $\alpha$ for combining attribute and relational similarity. For all of our experiments, we report the best accuracy over all values of $\alpha$ for each query. Selecting the optimal value of $\alpha$ for each query is an unresolved issue. However, our experiments reveal that even a fixed $\alpha(\alpha=0.5)$ for all queries brings significant improvements over the baselines.

The second issue is the determination of the termination threshold for RC-ER. Note that this is an issue for all of the baselines as well, and here we report best accuracy over all thresholds. This is an area of ongoing research. Preliminary experiments have shown that the best threshold is often query specific - setting the threshold depending on the ambiguity of the query results in significantly better accuracy than a fixed threshold for all queries. For an empirical evaluation, we cleaned the entire arXiv dataset offline by running RC-ER on all its references together, and terminated at the threshold that maximizes resolution accuracy over all references. This results in an overall accuracy (F1) of 0.98 . However, the average accuracy measured over the 75 queries in our test set is only 0.87 . In comparison, the best obtainable accuracy when resolving the queries individually each with a different threshold is 0.97 . This suggests that there may be potential benefits to localized cleaning over its global counterpart in the offline setting.

\section{Related Work}

The entity resolution problem has been studied in many different areas under different names - deduplication, record linkage, co-reference resolution, reference reconciliation, object consolidation, etc. Much of the work has focused on traditional attribute-based entity resolution. Extensive research has been done on defining approximate string similarity measures (Monge \& Elkan, 1996; Navarro, 2001; Bilenko et al., 2003; Chaudhuri, Ganjam, Ganti, \& Motwani, 2003) that may be used for unsupervised entity resolution. The other approach uses adaptive supervised algorithms that learn similarity measures from labeled data (Tejada, Knoblock, \& Minton, 2001; Bilenko \& Mooney, 2003).

Resolving entities optimally is known to be computationally hard even when only attributes are considered (Cohen, Kautz, \& McAllester, 2000). Therefore, efficiency has received a lot of attention in attribute-based data cleaning. The goal essentially is to avoid irrelevant and expensive attribute similarity computations using a 'blocking' approach without affecting accuracy significantly (Hernández \& Stolfo, 1995; Monge \& Elkan, 1997; McCallum et al., 2000). The merge/purge problem was posed by Hernández and Stolfo (1995) with efficient schemes to retrieve potential duplicates without resorting to quadratic complexity. They use a 'sorted neighborhood method' where an appropriate key is chosen for matching. Records are then sorted or grouped according to that key and potential matches are identified using a sliding window technique. However, some keys may be badly distorted so that their matches cannot be spanned by the window and such cases will not be retrieved. 
The solution they propose is a multi-pass method over different keys and then merging the results using transitive closure. Monge and Elkan (1997) combine the union find algorithm with a priority queue look-up to find connected components in an undirected graph. McCallum et al. (2000) propose the use of canopies to first partition the data into overlapping clusters using a cheap distance metric and then use a more accurate and expensive distance metric for those data pairs that lie within the same canopy. Chaudhuri et al. (2003) use an error tolerant index for data warehousing applications for probabilistically looking up a small set of candidate reference tuples for matching against an incoming tuple. This is considered 'probabilistically safe' since the closest tuples in the database will be retrieved with high probability. This is also efficient since only a small number of matches needs to be performed. Swoosh (Benjelloun, Garcia-Molina, Su, \& Widom, 2005) has recently been proposed as a generic entity resolution framework that considers resolving and merging duplicates as a database operator and the goal is to minimize the number of record-level and feature-level operations. An alternative approach is to reduce the complexity of individual similarity computations. Gravano, Ipeirotis, Koudas, and Srivastava (2003) propose a sampling approach to quickly compute cosine similarity between tuples for fast text-joins within an SQL framework. All of these approaches enable efficient data cleaning when only attributes of references are considered.

Many recently proposed approaches take relations into account for data integration (Ananthakrishna et al., 2002; Bhattacharya \& Getoor, 2004, 2005; Kalashnikov et al., 2005; Dong et al., 2005). Ananthakrishna et al. (2002) introduce relational deduplication in data warehouse applications where there is a dimensional hierarchy over the relations. Kalashnikov et al. (2005) enhance attribute similarity between an ambiguous reference and the many entity choices for it with relationship analysis between the entities, like affiliation and co-authorship. In earlier work, we have proposed different measures for relational similarity and a relational clustering algorithm for collective entity resolution using relationships (Bhattacharya \& Getoor, 2004, 2007). Dong et al. (2005) collectively resolve entities of multiple types by propagating relational evidences in a dependency graph, and demonstrate the benefits of collective resolution in real datasets. Long, Zhang, Wú, and Yu (2006) have proposed a model for general multi-type relational clustering, though it has not been applied specifically for entity resolution. They perform collective factorization over related matrices using spectral methods to identify the cluster space that minimizes distortion over relationships and individual features at the same time. All of these approaches that make use of relationships either for entity matching (where the domain entities are known) or entity resolution (where the underlying entities also need to be discovered) have been shown to increase performance significantly over the attribute-based solutions for the same problems. However, the price they pay is in terms of computational complexity that increases due to a couple of different reasons. Firstly, the number of potential matches increases when relationships are considered and individual similarity computations also become more expensive. Secondly, collective resolution using relationships necessitates iterative solutions that make multiple passes over the data. While some of these approaches have still been shown to be scalable in practice, they cannot be employed for query-time cleaning in a straight-forward manner.

The idea of multi-relational clustering also comes up in the Inductive Logic Programming (ILP) literature. Emde and Wettschereck (1996) have used multi-relational similarity for 
instance-based classification of representations in first order logic. They define the similarity of two objects, e.g., of two people, as a combination of the similarity of their attribute values, such as their age, weight, etc., and the similarity of the objects that they are related to, such as the companies they work for. This is similar to the naive relational similarity that we discussed earlier, except that the similarity of the connected objects is also defined recursively in terms of their connected objects. Kirsten and Wrobel (1998) have used this recursive relational similarity measure for agglomerative clustering of first order representations. While recursive comparison of neighbors is shown to be effective in terms of accuracy of results, the computational challenge is again a major drawback.

Probabilistic approaches that cast entity resolution as a classification problem have been extensively studied. The groundwork was done by Fellegi and Sunter (1969). Others (Winkler, 2002; Ravikumar \& Cohen, 2004) have more recently built upon this work. Adaptive machine learning approaches have been proposed for data integration (Sarawagi \& Bhamidipaty, 2002; Tejada et al., 2001), where active learning requires the user to label informative examples. Probabilistic models that use relationships for collective entity resolution have been applied to named entity recognition and citation matching (Pasula et al., 2003; McCallum \& Wellner, 2004; Li et al., 2005; Singla \& Domingos, 2004). These probabilistic approaches are superior to similarity-based clustering algorithms in that they associate a degree of confidence with every decision, and learned models provide valuable insight into the domain. However, probabilistic inference for collective entity resolution is not known to be scalable in practice, particularly when relationships are also considered. These approaches have mostly been shown to work for small datasets, and are significantly slower than their clustering counterparts.

Little work has been done in the literature for query-centric cleaning or relational approaches for answering queries, where execution time is as important as accuracy of resolution. Approaches have been proposed for localized evaluation of Bayesian networks (Draper \& Hanks, 1994), but not for clustering problems. Recently, Chandel, Nagesh, and Sarawagi (2006) have addressed efficiency issues in computing top-k entity matches against a dictionary in the context of entity extraction from unstructured documents. They process top-k searches in batches where speed-up is achieved by sharing computation between different searches. Fuxman, Fazli, and Miller (2005) motivate the problem of answering queries over databases that violate integrity constraints and address scalability issues in resolving inconsistencies dynamically at query-time. However, the relational aspect of the problem, which is the major scalability issue that we address, does not come up in any of these settings. In our earlier work on relational clustering(Bhattacharya \& Getoor, 2007), we used the idea of 'relevant references' for experimental evaluation on the BioBase dataset. As we have also discussed here, this dataset has entity labels only for the 100 most frequent names. Therefore, instead of running collective resolution over the entire BioBase dataset, we evaluated the 100 names separately, using only the 'relevant references' in each case. The relevant references were the ones directly connected to references having the names of interest. The concept of focused cleaning, the performance analysis of relational clustering, the expand-resolve strategy and, most importantly, the idea of adaptive expansion for query-time resolution were not addressed in that paper.

One of the first papers to make use of relational features for classification problem was by Chakrabarti, Dom, and Indyk (1998). They showed that for the problem of classifying 
hyper-linked documents, naive use of relationships can hurt performance. Specifically, if key terms from neighboring documents are thrown into the document whose topic is to be classified, classification accuracy degrades instead of improving. The parallel in our scenario of clustering using relationships is that the naive relational model (NR) may perform worse than the attribute model $(\mathbf{A})$ in the presence of highly ambiguous relationships. Chakrabarti et al. (1998) showed that relationships can however be used for improved classification when the topic labels of the neighboring documents are used as evidence instead of naively considering the terms that they contain. In our earlier work (Bhattacharya \& Getoor, 2004, 2007), we have shown similar results for collective clustering using relationships, where the cluster labels of neighboring labels lead to improved clustering performance compared to naive relational and attribute-based clustering. The interesting result that we have shown in this paper both in theory and empirically is that even collective use of relationships can hurt clustering accuracy compared to attribute-based clustering. This happens when relationships between references are dense and ambiguous, and errors that propagate over relationships exceed the identifying evidence that they provide.

\section{Conclusions}

In this paper, we have motivated the problem of query-time entity resolution for accessing unresolved third-party databases. For answering entity resolution queries, we have addressed the challenges of using collective approaches, which have recently shown significant performance improvements over traditional baselines in the offline setting. The first hurdle for collective resolution arises from the interdependent nature of its resolution decisions. We first formally analyzed the recursive nature of this dependency, and showed that the precision and recall for individual entities grow in a geometric progression as increasing levels of neighbors are considered and collectively resolved. We then proposed a two-stage 'expand and resolve' strategy for answering queries based on this analysis, using two novel expansion operators. We showed using our analysis that it is sufficient to consider neighbors up to small expansion depths, since resolution accuracy for the query converges quickly with increasing expansion level. The second challenge for answering queries is that the computation has to be quick. To achieve this, we improved on our unconstrained expansion strategy to propose an adaptive algorithm, which dramatically reduces the size of the relevant references and, as a result, the processing time - by identifying the most informative references for any query. We demonstrated using experiments on two real datasets that our strategies enable collective resolution at query-time, without compromising on accuracy. We additionally performed various experiments on synthetically generated data over a wide range of settings to verify the trends predicted by our analysis. In summary, we have addressed and motivated a critical data integration and retrieval problem, proposed algorithms for solving it accurately and efficiently, provided a theoretical analysis to validate our approach and explain why it works, and, finally, shown experimental results on multiple real-world and synthetically generated datasets to demonstrate that it works extremely well in practice. While we have presented results for bibliographic data, the techniques are applicable in other relational domains.

While we have shown the dramatic reduction in query processing time that comes with adaptive expansion, more research is necessary to be able to answer entity resolution queries 
on the order of milli-seconds, as may be demanded in many scenarios. Interesting directions of future research include exploring stronger coupling between the extraction and resolution phases of query processing, where the expansion happens "on-demand" only when the resolution process finds the residual ambiguity to be high and requires additional evidence for taking further decisions. This would directly address the problem of determining the expansion depth. While we have reported some preliminary experiments in this paper, more work needs to be done on adaptive depth determination depending on ambiguity. In the same context, we may imagine "soft" thresholds for adaptive expansion, where the expansion operator automatically determines the number of hyper-edges or names to be expanded so that the residual ambiguity falls below some specified level. Other interesting extensions include caching of intermediate resolutions, where the related resolutions performed for any query are stored and retrieved as and when required for answering future queries.

\section{Acknowledgments}

We wish to thank our anonymous reviewers for their constructive suggestions which greatly improved this paper. This work was supported by the National Science Foundation, NSF \#0423845 and NSF \#0438866, with additional support from the ITIC KDD program.

\section{Appendix A}

\section{Synthetic Data Generator}

We have designed a synthetic data generator that allows us to control different structural and attribute-based characteristics of the data(Bhattacharya \& Getoor, 2007). Here we present an overview of the generation algorithm.

The generation process has two stages. In the first stage, we create the collaboration graph among the underlying entities and the entity attributes. In the second, we generate observed co-occurrence relations from this collaboration graph. A high level description of the generative process in shown in Figure 10. Next, we describe the two stages of the generation process in greater detail.

The graph creation stage, in turn, has two sub-stages. First, we create the domain entities and their attributes and then add relationships between them. For creating entities, we control the number of entities and the ambiguity of their attributes. We create $N$ entities and their attributes one after another. For simplicity and without losing generality, each entity $e$ has a single floating point attribute $e . x$, instead of a character string. A parameter $p_{a}$ controls the ambiguity of the entity attributes; with probability $p_{a}$ the attribute of a new entity is chosen from values that are already in use by existing entities. Then $M$ binary relationships are added between the created entities. As with the attributes, there is a parameter controlling the ambiguity of the relationships, as defined in Section 4. For each binary relationship $\left(e_{i}, e_{j}\right)$, first $e_{i}$ is chosen randomly and then $e_{j}$ is sampled so that $\left(e_{i}, e_{j}\right)$ is an ambiguous relationship with probability $p_{a}^{R}$.

Before describing the process of generating co-occurrence relationships from the graph, let us consider in a little more detail the issue of attribute ambiguity. What finally needs to be controlled is the ambiguity of the reference attributes. While these depend on the entity attributes, they are not completely determined by entities. Taking the example 


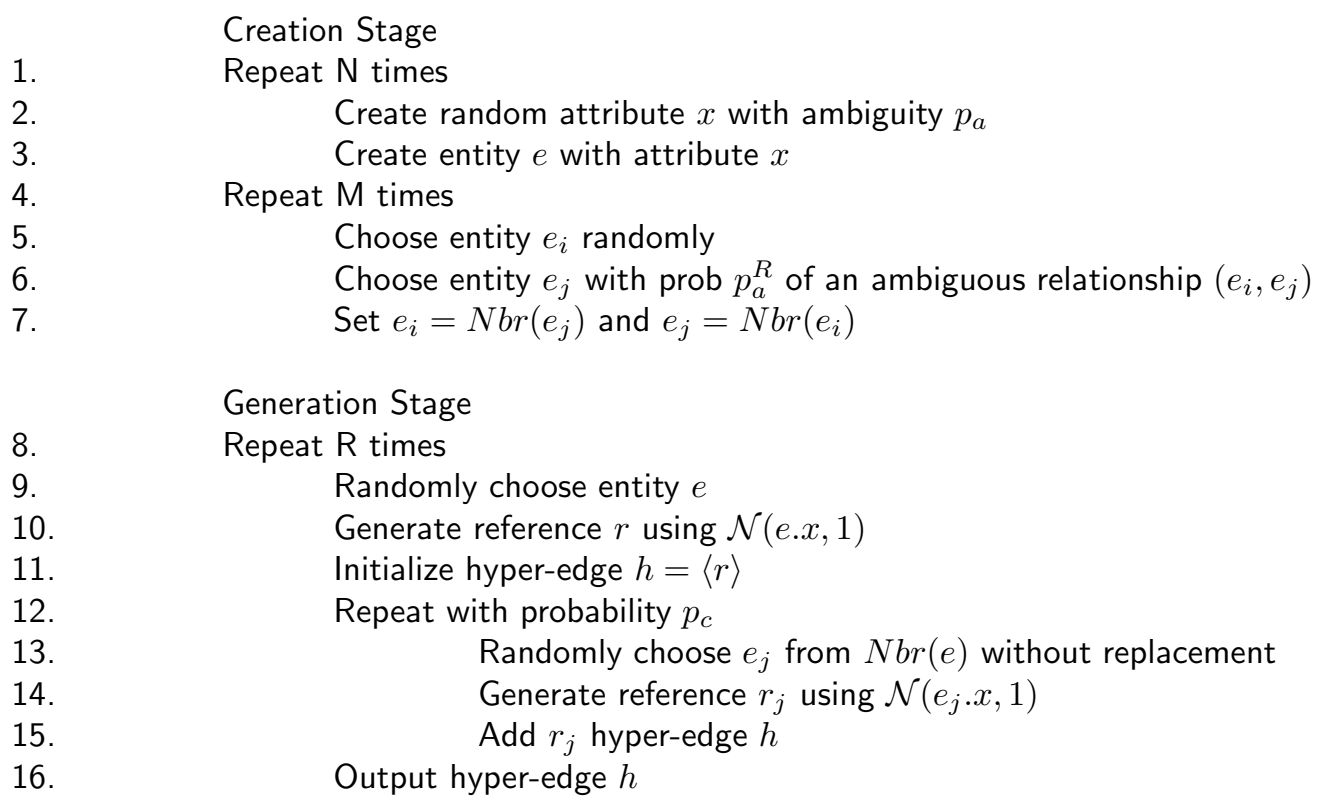

Figure 10: High-level description of synthetic data generation algorithm

of names, two people who have names 'John Michael Smyth' and 'James Daniel Smith' can still be ambiguous in terms of their observed names in the data depending on the generation process of observed names. In other words, attribute ambiguity of the references depends both on the separation between entity attributes and the dispersion created by the generation process. We make the assumption that for an entity $e$ with attribute $e . x$, its references are generated from a Gaussian distribution with mean $x$ and variance 1.0. So, with very high probability, any reference attribute generated from $e . x$ will be in the range $[e . x-3, e . x+3]$. So this range in the attribute domain is considered to be 'occupied' by entity $e$. Any entity has an ambiguous attribute if its occupied range intersects with that of another entity.

Now we come to the generation of co-occurrence relationships from the entity collaboration graph. In this stage, $R$ co-occurrence relationships or hyper-edges are generated, each with its own references. For each hyper-edge $\left\langle r_{i}, r_{i 1}, \ldots, r_{i k}\right\rangle$, two aspects need to be controlled - how many references and which references should be included in this hyper-edge. This is done as follows. First, we sample an entity $e_{i}$ which serves the initiator entity for this hyper-edge. Then other entities $e_{i j}$ for this hyper-edge are repeatedly sampled (without replacement) from the neighbors of the initiator entity $e_{i}$. The size of the hyper-edge is determined using a parameter $p_{c}$. The sampling step for a hyper-edge is terminated with probability $p_{c}$ after each selection $e_{i j}$. The process is also terminated when the neighbors of the initiator entity are exhausted. Finally, references $r_{i j}$ need to be generated from each of the selected entities $e_{i j}$. This is done for each entity $e$ by sampling from its Gaussian distribution $\mathcal{N}(e . x, 1)$. 


\section{References}

Ananthakrishna, R., Chaudhuri, S., \& Ganti, V. (2002). Eliminating fuzzy duplicates in data warehouses. In The International Conference on Very Large Databases (VLDB), Hong Kong, China.

Benjelloun, O., Garcia-Molina, H., Su, Q., \& Widom, J. (2005). Swoosh: A generic approach to entity resolution. Tech. rep., Stanford University.

Bhattacharya, I., \& Getoor, L. (2004). Iterative record linkage for cleaning and integration. In The SIGMOD Workshop on Research Issues on Data Mining and Knowledge Discovery (DMKD), Paris, France.

Bhattacharya, I., \& Getoor, L. (2005). Relational clustering for multi-type entity resolution. In The ACM SIGKDD Workshop on Multi Relational Data Mining (MRDM), Chicago, IL, USA.

Bhattacharya, I., \& Getoor, L. (2006). Mining Graph Data (L. Holder and D. Cook, eds.), chap. Entity Resolution in Graphs. Wiley.

Bhattacharya, I., \& Getoor, L. (2007). Collective entity resolution in relational data. ACM Transactions on Knowledge Discovery from Data (TKDD), 1(1).

Bhattacharya, I., Licamele, L., \& Getoor, L. (2006). Query-time entity resolution. In The ACM International Conference on Knowledge Discovery and Data Mining (SIGKDD), Philadelphia, PA, USA.

Bilenko, M., \& Mooney, R. (2003). Adaptive duplicate detection using learnable string similarity measures. In The ACM International Conference on Knowledge Discovery and Data Mining (SIGKDD), Washington DC, USA.

Bilenko, M., Mooney, R., Cohen, W., Ravikumar, P., \& Fienberg, S. (2003). Adaptive name matching in information integration.. IEEE Intelligent Systems, 18(5), 16-23.

Chakrabarti, S., Dom, B., \& Indyk, P. (1998). Enhanced hypertext categorization using hyperlinks. In Proceedings of the ACM International Conference on Management of Data (SIGMOD).

Chandel, A., Nagesh, P. C., \& Sarawagi, S. (2006). Efficient batch top-k search for dictionary-based entity recognition. In The IEEE International Conference on Data Engineering (ICDE), Washington, DC, USA.

Chaudhuri, S., Ganjam, K., Ganti, V., \& Motwani, R. (2003). Robust and efficient fuzzy match for online data cleaning. In The ACM International Conference on Management of Data (SIGMOD), San Diego, CA, USA.

Cohen, W., Kautz, H., \& McAllester, D. (2000). Hardening soft information sources. In The ACM International Conference on Knowledge Discovery and Data Mining (SIGKDD), Boston, MA, USA.

Dong, X., Halevy, A., \& Madhavan, J. (2005). Reference reconciliation in complex information spaces. In The ACM International Conference on Management of Data (SIGMOD), Baltimore, MD, USA. 
Draper, D., \& Hanks, S. (1994). Localized partial evaluation of belief networks. In The Annual Conference on Uncertainty in Artificial Intelligence (UAI), Seattle, WA, USA.

Emde, W., \& Wettschereck, D. (1996). Relational instance based learning. In Proceedings of the International Conference on Machine Learning (ICML).

Fellegi, I., \& Sunter, A. (1969). A theory for record linkage. Journal of the American Statistical Association, 64, 1183-1210.

Fuxman, A., Fazli, E., \& Miller, R. (2005). Conquer: Efficient management of inconsistent databases. In The ACM International Conference on Management of Data (SIG$M O D)$, Baltimore, MD, USA.

Gravano, L., Ipeirotis, P., Koudas, N., \& Srivastava, D. (2003). Text joins for data cleansing and integration in an rdbms. In The IEEE International Conference on Data Engineering (ICDE), Bangalore, India.

Hernández, M., \& Stolfo, S. (1995). The merge/purge problem for large databases. In The ACM International Conference on Management of Data (SIGMOD), San Jose, CA, USA.

Kalashnikov, D., Mehrotra, S., \& Chen, Z. (2005). Exploiting relationships for domainindependent data cleaning. In SIAM International Conference on Data Mining (SIAM $S D M)$, Newport Beach, CA, USA.

Kirsten, M., \& Wrobel, S. (1998). Relational distance-based clustering. In Proceedings of the International Workshop on Inductive Logic Programming (ILP).

Li, X., Morie, P., \& Roth, D. (2005). Semantic integration in text: From ambiguous names to identifiable entities. AI Magazine. Special Issue on Semantic Integration, 26(1).

Long, B., Zhang, Z. M., Wú, X., \& Yu, P. S. (2006). Spectral clustering for multi-type relational data. In Proceedings of the 23rd International Conference on Machine Learning $(I C M L)$.

McCallum, A., Nigam, K., \& Ungar, L. (2000). Efficient clustering of high-dimensional data sets with application to reference matching. In The ACM International Conference On Knowledge Discovery and Data Mining (SIGKDD), Boston, MA, USA.

McCallum, A., \& Wellner, B. (2004). Conditional models of identity uncertainty with application to noun coreference. In Advances In Neural Information Processing Systems (NIPS), Vancouver, BC, Canada.

Monge, A., \& Elkan, C. (1996). The field matching problem: Algorithms and applications. In The ACM International Conference on Knowledge Discovery and Data Mining (SIGKDD), Portland, OR, USA.

Monge, A., \& Elkan, C. (1997). An efficient domain-independent algorithm for detecting approximately duplicate database records. In The SIGMOD Workshop on Research Issues on Data Mining and Knowledge Discovery (DMKD), Tuscon, AZ, USA.

Navarro, G. (2001). A guided tour to approximate string matching. ACM Computing Surveys, 33(1), 31-88. 
Pasula, H., Marthi, B., Milch, B., Russell, S., \& Shpitser, I. (2003). Identity uncertainty and citation matching. In Advances in Neural Information Processing Systems (NIPS), Vancouver, BC, Canada.

Ravikumar, P., \& Cohen, W. (2004). A hierarchical graphical model for record linkage. In The Conference on Uncertainty in Artificial Intelligence (UAI), Banff, Alberta, Canada.

Sarawagi, S., \& Bhamidipaty, A. (2002). Interactive deduplication using active learning. In Proceedings of the Eighth ACM International Conference on Knowledge Discovery and Data Mining (SIGKDD), Edmonton, Alberta, Canada.

Singla, P., \& Domingos, P. (2004). Multi-relational record linkage. In The SIGKDD Workshop on Multi-Relational Data Mining (MRDM), Seattle, WA, USA.

Tejada, S., Knoblock, C., \& Minton, S. (2001). Learning object identification rules for information integration. Information Systems Journal, 26(8), 635-656.

Winkler, W. (2002). Methods for record linkage and Bayesian networks. Tech. rep., Statistical Research Division, U.S. Census Bureau, Washington, DC. 